OPEN ACCESS

Edited by:

Qiang Wei,

Sichuan University, China

Reviewed by:

Jennifer Young,

Max Planck Institute for Medical Research (MPIMF), Germany

Silviya Petrova Zustiak,

Saint Louis University, United States

*Correspondence: Jung Yul Lim jlim4@unl.edu

Specialty section:

This article was submitted to Biomaterials,

a section of the journal Frontiers in Bioengineering and Biotechnology

Received: 21 September 2020 Accepted: 22 December 2020 Published: 18 January 2021

Citation:

Riehl BD, Kim E, Bouzid T and Lim JY (2021) The Role of Microenvironmental Cues and Mechanical Loading Milieus in Breast Cancer Cell Progression and

Metastasis.

Front. Bioeng. Biotechnol. 8:608526. doi: 10.3389/fbioe.2020.608526

\section{The Role of Microenvironmental Cues and Mechanical Loading Milieus in Breast Cancer Cell Progression and Metastasis}

\author{
Brandon D. Riehl ${ }^{1}$, Eunju Kim ${ }^{1}$, Tasneem Bouzid ${ }^{1}$ and Jung Yul Lim ${ }^{1,2 \star}$ \\ ${ }^{1}$ Department of Mechanical and Materials Engineering, University of Nebraska-Lincoln, Lincoln, NE, United States, ${ }^{2}$ Mary \\ and Dick Holland Regenerative Medicine Program, University of Nebraska Medical Center, Omaha, NE, United States
}

Cancer can disrupt the microenvironments and mechanical homeostatic actions in multiple scales from large tissue modification to altered cellular signaling pathway in mechanotransduction. In this review, we highlight recent progresses in breast cancer cell mechanobiology focusing on cell-microenvironment interaction and mechanical loading regulation of cells. First, the effects of microenvironmental cues on breast cancer cell progression and metastasis will be reviewed with respect to substrate stiffness, chemical/topographic substrate patterning, and 2D vs. 3D cultures. Then, the role of mechanical loading situations such as tensile stretch, compression, and flow-induced shear will be discussed in relation to breast cancer cell mechanobiology and metastasis prevention. Ultimately, the substrate microenvironment and mechanical signal will work together to control cancer cell progression and metastasis. The discussions on breast cancer cell responsiveness to mechanical signals, from static substrate and dynamic loading, and the mechanotransduction pathways involved will facilitate interdisciplinary knowledge transfer, enabling further insights into prognostic markers, mechanically mediated metastasis pathways for therapeutic targets, and model systems required to advance cancer mechanobiology.

Keywords: breast cancer, metastasis, substrate microenvironments, mechanical loading, mechanotransduction, cell progression

\section{INTRODUCTION}

Breast cancer is the most diagnosed cancer among women in the US with significant mortality resulting from metastasis (DeSantis et al., 2019). Early detection and prevention of breast cancer metastasis could greatly improve patient outcomes especially for cases when breast cancer migrates to crucial organs including brain, liver, lung, and bone. Recently, mechanical factors are becoming more and more recognized as important regulators of oncogenesis, tumor progression, and metastasis. The mechanical factors may originate from microenvironments, e.g., extracellular matrix (ECM) stiffness, topography, composition, etc., as well as from dynamic mechanical loading situations, e.g., tensile strain, compression, flow-induced shear, etc. Microenvironmental factors at the premetastatic niche may prime tumor cells toward invasive phenotypes causing a host of genetic and mechanical adaptations, which then can feedback to reinforce the aberrant ECM environment. Further adaptations accrue when tumor cells proliferate in a confined and stiffened ECM milieu 
TABLE 1 | Some representative concepts/findings in microenvironmental cues.

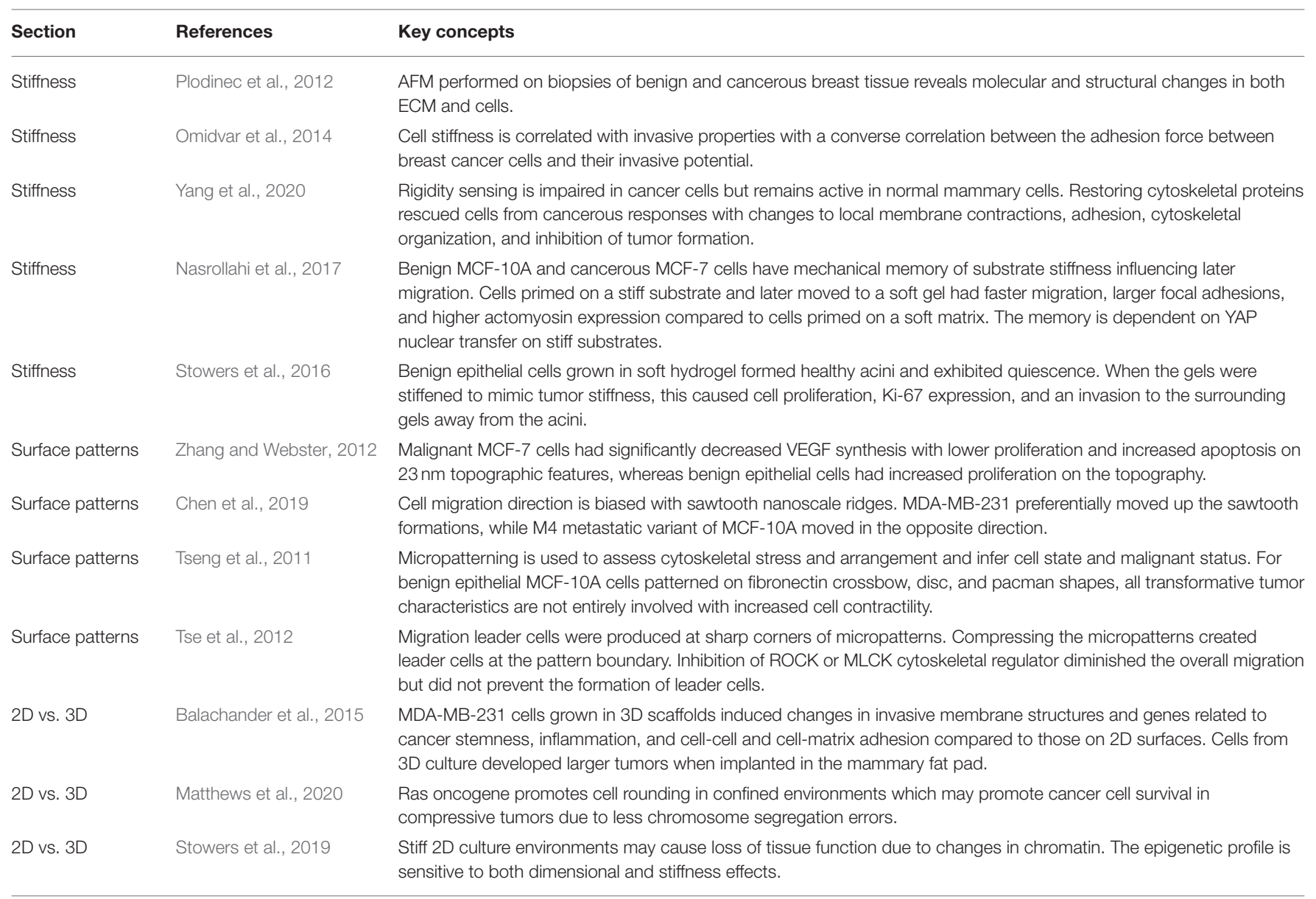

causing a rise in solid stress within the tumor. As the vasculature in the tumor is remodeled, cells also experience elevated interstitial pressure and thus compressive effect. Moreover, tensile stress may be experienced at the tumor periphery, and interstitial outflow is becoming developed. In the next step, metastasizing cells are exposed to fluid flow-induced shear stress during distal migration through the lymph and vascular systems. Tumor cell migration through capillaries and intravasation and extravasation induce compression and large cellular deformations in the form of constrictions. Finally, each metastasis target site has unique microenvironmental and dynamic mechanical loading signals which may contribute to disease progression or induce dormancy. Here, we will first review the updated understanding on the effects of key microenvironmental cues on breast cancer cell behavior (the representative findings on microenvironments are highlighted in Table 1). The effects of dynamic mechanical loading environments on breast cancer cells will then be described including those at the distal metastasis site (representative findings on mechanical loading cues are highlighted in Table 2). Then, the discussion of potential regulatory cellular mechanotransduction mechanisms involved in the mechanical factor regulation of breast cancer cells is provided before ending with a perspective. Note, for detailed classification of breast cancer cell lines described in this review, the works by Neve et al. (2006) and Holliday and Speirs (2011) are referred.

\section{EFFECTS OF MICROENVIRONMENTAL CUES}

\section{Substrate Stiffness}

It has been recognized that the composition, mechanical stiffness, and dimensionality of cancerous extracellular environments greatly influence cancer cell physiology and progression. Specifically, the alteration in tissue stiffness indicates one of the earliest indicators of cancer presence. Forces between cells and cell and ECM are dependent on the increase in breast tissue stiffness which is associated with the risk of breast cancer. This may be assessed radiologically with dense breast tissue having an increase in cell number, collagen fibers, and proteoglycans compared to breast tissue with low mammographic density (Boyd et al., 2014). Cancers have molecular and structural changes in both the ECM and cells. Such alteration is evident in atomic force microscopy (AFM) performed on biopsies of benign and cancerous breast tissues (Plodinec et al., 2012). Tissues from benign biopsies had uniform stiffness with a single peak 
TABLE 2 | Some representative concepts/findings in mechanical loading milieus.

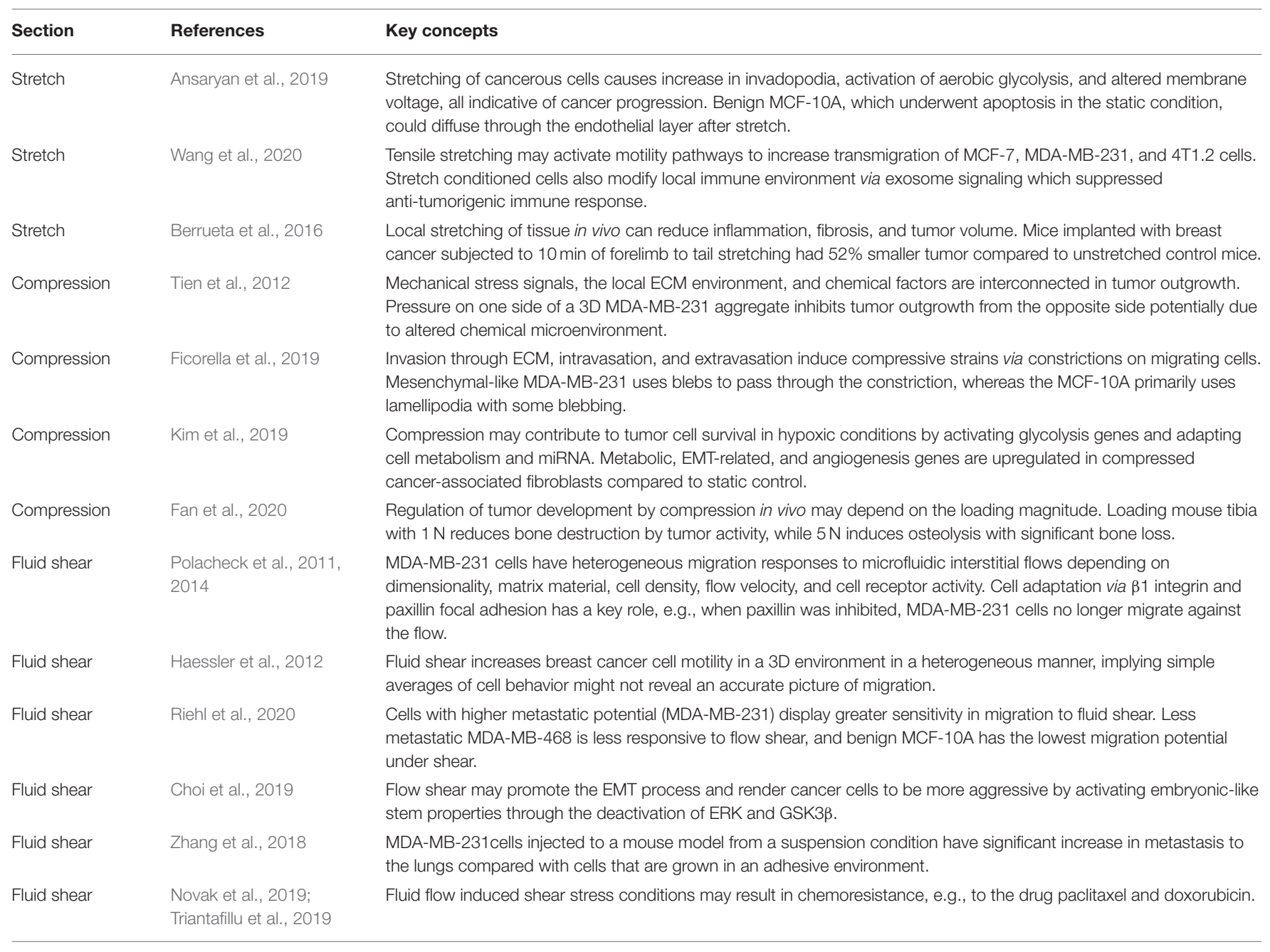

in the correlative stiffness map, while malignant tissues showed heterogeneous stiffness with a broad distribution in correlative stiffness maps and a low-stiffness peak due to soft cancer cells. The differences in normal, tumorigenic, and metastatic cells have been demonstrated in another study by microrheology confirming cancerous cells are significantly softer than benign counterparts (Smelser et al., 2015). The surrounding tissues remodel and adapt in response to cancer growth resulting in aberrant ECM moduli. This can then work as a feedback loop since cancerous cells on abnormal stiffness have responses that may further cancer progression.

The changes in tissue stiffness have been correlated with breast cancer invasion and aggression. When cancer transforms the ECM, additional collagen is deposited and the local ECM at the tumor invasive front is linearized. Correlating this to invasive cell behavior, the ECM was stiffest and most heterogeneous around the aggressive basal-like, HER2+ tumors compared with less aggressive luminal tumors (Acerbi et al., 2015). The stiff matrix can cause alterations in cell adhesion structures and dynamics. For instance, the stiff matrix may induce a mesenchymal-like cell phenotype which is evident in the focal adhesion assemblies and forces produced, and such changes may be key to cancer cell invasion and migration in confined spaces (Mekhdjian et al., 2017). Interfaces to the cells are also altered in cancer with significant changes to glycocalyx cell coating in tumor cells contributing to malignancy. The glycocalyx coating can amplify mechanical signals on the cell surface and modify transmembrane protein expressions. For example, the expression of transmembrane mechanical linkage protein, integrin, is altered in cancer cells when large glycoproteins are present in the glycocalyx (Paszek et al., 2014). These stiffness adaptations can have, eventually, implications for the efficacy of cancer treatment. The efficiency of the therapeutic lapatinib which acts on the HER2 pathway in cancer cells was found to be modulated by microenvironment mechanical properties: HCC1569 breast cancer cells were responsive to lapatinib on a collagen gel with $400 \mathrm{~Pa}$ stiffness but this response was absent on the tissue culture plastic with $>2$ GPa stiffness (Lin et al., 2015).

In addition to altered ECM stiffness, as mentioned above, cancer cells themselves can also have altered stiffness. A converse 
correlation was found between the stiffness of the cancer cells and their invasive properties: in the order of invasiveness from less to more, the average stiffness as measured by AFM was $1.05,0.94$, and $0.62 \mathrm{kPa}$ for MCF-7, T47D, and MDA-MB-231 cells, respectively (Omidvar et al., 2014). It has been established that breast cancer cells cultured on stiff surfaces have in general higher proliferation, higher migration velocity, and increased chemoresistance compared with those grown on soft substrates (McGrail et al., 2015). Furthermore, breast cancer cells showed a preference for stiffer surfaces by exhibiting durotaxis (i.e., migration from soft to stiff region) on polyacrylamide stiffness gradients (DuChez et al., 2019). Cells showed durotaxis with migration distance dependent on wherein the cells were seeded: cells starting in softer regions migrated further than cells seeded on medium or stiff regions of the gradient. This provides evidence that cancer cells can sense variations in the ECM stiffness as may be found in heterogeneous tumors or at tumor boundaries.

Via testing the deformation of sub- $\mu \mathrm{m}$ scale polydimethylsiloxane (PDMS) micropillars by the cells, it was found that substrate rigidity sensing was impaired in metastatic MDA-MB-231 cells but remained active in normal MCF-10A mammary cells (Wolfenson et al., 2016). As a mechanism, the rigidity sensing could be restored via tropomyosin 2.1 expression, or the normal cells could transform to a cancerous phenotype with tropomyosin 2.1 inhibition. In a follow up study (Yang et al., 2020), restoring cytoskeletal proteins rescued cells from cancerous responses with changes to local membrane contraction, adhesion, cytoskeletal organization, and as a result inhibited tumor formation. Moreover, inhibiting cytoskeletal elements including myosin IIA reinforced cancer phenotypes. Translating these results into an in vivo model, MDA-MB231 cells with rigidity sensing restored by YFP-tropomyosin 2.1 transfection had less tumorigenic responses in a chick chorioallantoic model compared to green fluorescent protein (GFP) transfected control. The MDA-MB-231 cells with tropomyosin expression formed tumors with $36.5 \%$ lower tumor weight and the tumor volume decreased over time, whereas the control MDA-MB-231 cells had increased tumor volume with time. Taken together, these results demonstrate that cancerous cells have lower stiffness and fewer rigidity sensing mechanisms compared to normal cells.

Migration out of the premetastatic niche exposes tumor cells to new ECM mechanical environments which may affect cancer progression. Such environmental changes likely function through specific ECM ligand and integrin interaction. For example, abnormally high stiffness, as can be seen in the bone metastatic milieu, can regulate bone-destructive phenotype in MDA-MB231 cells via regulating specific integrin subunit (Page et al., 2015). It was found that parathyroid hormone-related protein (PTHrP), a factor associated with tumor destruction of bone, and integrin $\beta 3$ expression showed increases with increasing substrate rigidity. Taking advantage of this, injection of MDA-MB-231 cells with blocked $\beta 3$ integrin did not show tumor-induced bone destruction, providing a potential therapeutic target. Further down this mechanosignaling pathway, Src, PI3K, Rac1, and rho-associated kinase (ROCK) inhibitors were tested for the regulation of general invasive epithelial phenotype (Carey et al.,
2017). Inhibiting Src and ROCK promoted invasive protrusions, whereas inhibiting PI3K and Racl suppressed invasion. When testing the stiffening of the ECM, adding stiffer collagen caused benign MCF-10A to have an invasive phenotype and promoted cell dispersion. Unlike culture in Matrigel which promotes healthy epithelial formation, stiffer collagen matrix caused an invasive epithelial phenotype with activated mesenchymal genes and proteins including vimentin, fibronectin, and Snail (Figure 1) (Carey et al., 2017).

Even if tumor cells are removed, the remaining cells may have been primed with the mechanical memory effect. Benign epithelial MCF-10A and cancerous MCF-7 cells displayed the mechanical memory effect influencing later migration capability (Nasrollahi et al., 2017). Cells that were seeded on a stiff polyacrylamide substrate and later moved to a soft gel had faster migration, larger focal adhesions, and higher actomyosin expression compared to cells that were primed on a soft matrix. This mechanical memory was dependent on yes-associated protein (YAP), which was translocated and retained inside the nucleus when cultured on stiff substrates, and inhibiting YAP significantly suppressed the memory-dependent migration. This provides evidence that epithelial cells can have lasting effects from local tissue stiffening. The mechanical memory effect may also exist in cells associated with the epithelial cells, e.g., cancerassociated fibroblasts. Primary fibroblasts isolated from tumors exerted higher traction force and had higher migration than fibroblasts from non-cancerous sources (Alcoser et al., 2015). In $3 \mathrm{D}$ collagen matrix, fibroblasts from breast tumors migrated twice as far as fibroblasts from non-cancerous regions, suggesting that cancer must be studied as a combined system and studying only tumor cells may not provide a thorough picture.

As suggested above, recently, a particular interest in the memory effect of substrate stiffness includes YAP. The results with in vitro culture can be further supported with the tissue and in vivo mouse models. It was observed that initiation of metastatic colonization by organ-specific MDA-MB-231 (which is brain, bone, and lung metastatic) in capillaries was achieved via activated YAP (Er et al., 2018). Disseminated MDA-MB231 cells used cell adhesion molecule L1 (L1CAM) to spread on capillaries, thus activating YAP via $\beta 3$ integrin expression. The resultant replacement of local pericytes by cancer cells drove the early metastatic invasion. Similarly, cancer associated fibroblasts (CAFs) may require YAP mechanotransduction for formation and maintenance. In fibroblasts collected from various stages of cancer progression, feedforward regulation was found in which YAP regulates cytoskeletal tension and increasing this tension causes further elevation of YAP (Calvo et al., 2013). Inhibiting ROCK could prevent this feedforward loop, reversing the cancer-associated fibroblast phenotype. These mechanisms may not be limited to interactions between tumor cells and fibroblasts, but also have been noted in a two-way regulation in mesenchymal stem cell (MSC) differentiation to CAFs by factors from mammary cancer cells and CAFs in turn promoting cancer development. Specifically, soluble factors from cancer cells caused MSC differentiation to CAFs on stiff surfaces, but this CAF-forming mechanism was lacking when MSCs were seeded on soft substrates (Ishihara et al., 2017). It was suggested that 
A
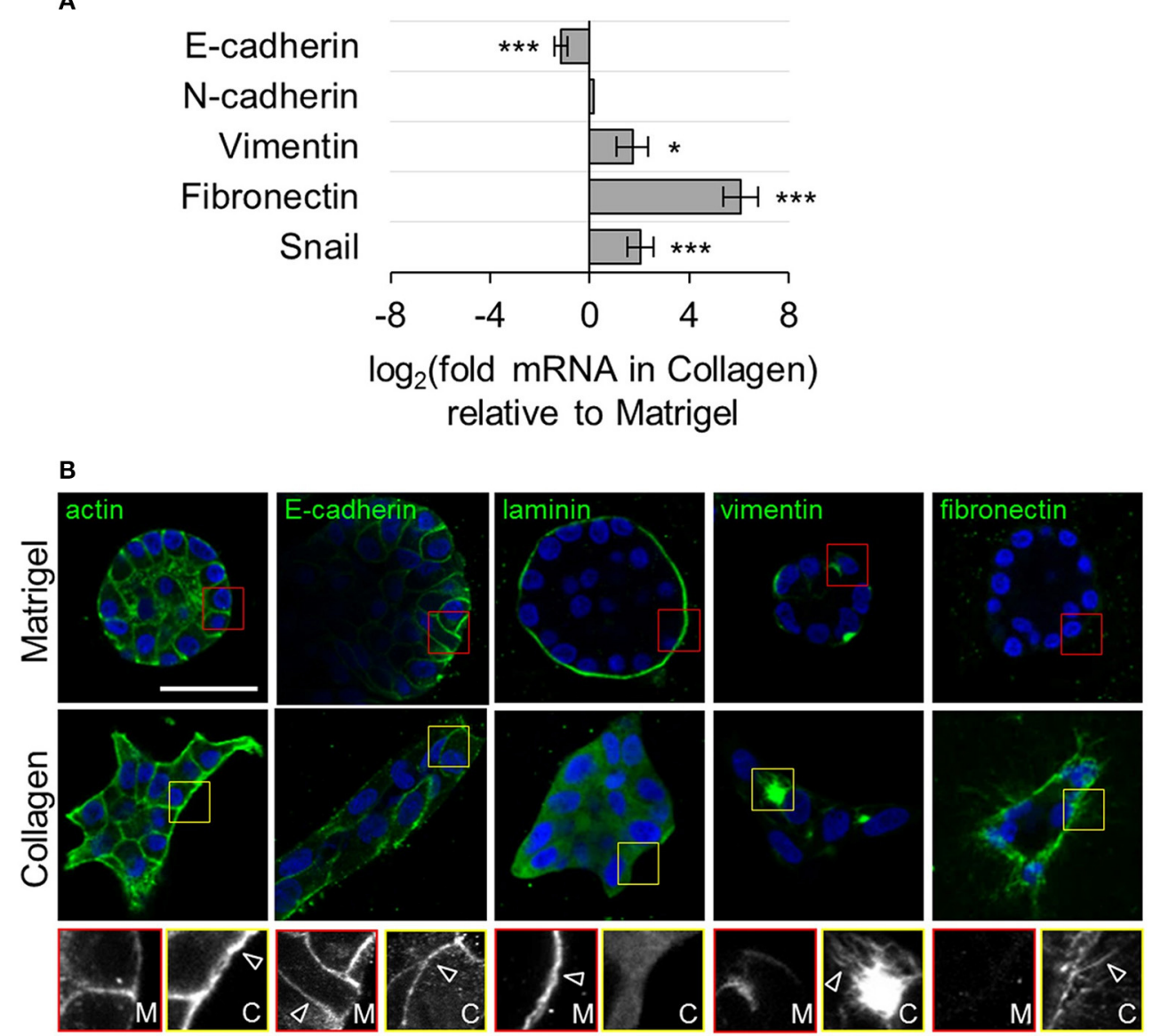

FIGURE 1 | Benign epithelial MCF-10A have mesenchymal-like gene expression and invasive morphology when grown in a matrix that mimics tumor environments. Matrigel was used as a control that mimicked the properties of healthy breast tissue and collagen mimicked a cancerous scaffold. (A) E-cadherin was downregulated and mesenchymal markers vimentin, fibronectin, and snail were upregulated in collagen. ${ }^{\star} p=0.01$, ${ }^{* \star *} p<0.0001$. (B) Staining of actin, E-cadherin, and

mesenchymal markers with confocal imaging revealed normal acini structures in matrigel and protrusive and invasive structures in collagen. Regions of interest are in red for matrigel and yellow for collagen with arrows marking notable features. Scale bar is $50 \mu \mathrm{m}$. Reprinted with permission from Springer Nature (Carey et al., 2017).

MSC promotion of tumors is partially dependent on local tissue stiffness which acted via YAP and myosin light chain.

Development and maintenance of cancer stem cells may depend on the physical characteristics of the tumor microenvironment. Studies have screened substrate stiffness to determine the condition for the maintenance of cancer stem cell populations. It was observed that cancer stem cell marker expressions in MCF-7 and MDA-MB-231 were most prevalent at substrate stiffness of $5 \mathrm{kPa}$ compared to nonoptimal substrate stiffness of 2, 25, 50, and $70 \mathrm{kPa}$ (Jabbari et al., 2015). Moreover, growing cells on an unphysiological stiffness of around $1 \mathrm{kPa}$, similar to tumor stiffness, caused tumor-like development compared with cells grown on soft native mammary tissue stiffness in the range of $150 \mathrm{~Pa}$ (Stowers et al., 2016). In the study, MCF-10A cells grown in soft hydrogel for 14 days formed healthy acini and exhibited quiescence. When the gels were stiffened after 14 days to mimic tumor stiffness, this caused cell proliferation, Ki-67 expression, and an invasion to the surrounding gels away from the acini. Examining mechanotransduction inhibitors evidenced that inhibiting PI3K/Rac1 pathway decreased the size and number of invasive acini. Similarly, Wei et al. (2015) showed Eph4Ras and MCF-10A established ductal acini and did not invade the basement membrane when grown on polyacrylamide gels that mimicked the natural $150 \mathrm{~Pa}$ stiffness of native tissue. However, when grown on $5.7 \mathrm{kPa}$ gel that mimicked the stiffness of tumor environments, cells exhibited epithelial-mesenchymal transition (EMT) causing the invasion of the basement membrane. As a mechanism, the knockdown of TWIST1 could prevent the invasion of the basement membrane on stiff gels. TWIST1 was found to translocate from the cytoplasm to the nucleus on a rigid matrix for MCF-10A, MCF-10DCIS, Bt-549, and Eph4Ras cells, and blocking $\beta 1$ integrin inhibited the invasion and prevented TWIST1 nuclear translocation.

Going beyond cytoskeletal tension and measures of invasion, the scaffold stiffness likely influences many other processes 
including microvesicle (MV) release and cell metabolism. Cancer cell derived MVs may contribute to remodeling of the surrounding environment, drive remodeling in the local premetastatic niche, and possibly primes distal sites for invasion. For example, MVs released from MDA-MB-231 cells activated fibroblast proliferation, contractility, and spreading in a matrixstiffness dependent manner (Schwager et al., 2019). When MVs were applied to fibroblasts on substrates with the stiffness of healthy breast tissue, no changes were observed. However, when MVs were applied to fibroblasts on stiffer substrates mimicking tumorigenic breast tissue, MVs promoted spreading, cancer associated fibroblast-like phenotype, and contraction. In contrast, MVs from benign MCF-10A cells did not activate the cancer associated fibroblast behaviors on any substrates.

Besides substrate stiffness and related mechanical signaling to affect cancer cell behavior, exploring matrix composition and architecture may also be necessary. It was reported that cell energetics as measured by the ATP:ADP ratio depended on the matrix composition and the extent to which the matrix facilitated migration, e.g., ATP:ADP ratio in MDA-MB-231 was increased on cell migration impairing denser matrices, but decreased on migration facilitating matrices composed of aligned collagens (Zanotelli et al., 2018). The result is relevant for the tradeoff of leader and follower cells in that the coordinated relay-style tradeoff can be regulated by the energy state of the leader cells (Zhang et al., 2019).

For investigating breast cancer metastasis to soft tissues such as brain, soft matrices are particularly needed. Brain metastatic MDA-MB-231Br grown on hyaluronic hydrogels with a range of physiologically relevant, brain-mimicking stiffness displayed significant increases in spreading, adhesion, proliferation, and migration with increasing stiffness (Narkhede et al., 2018). Increased migration speed in particular depended on welldeveloped actin cytoskeleton on the stiff matrix. As a related governing mechanism, blocking focal adhesion kinase (FAK) on stiff hydrogels suppressed the cell adhesion, proliferation, and migration responses. Building on these, in a subsequent study, brain metastatic MDA-MB-231Br and BT-474Br3 cells were tested for dormancy on gels with varying stiffness (Narkhede et al., 2020). Brain metastatic breast cancer cells were dormant, as assessed by lower EdU and Ki67, when seeded on soft (0.4 $\mathrm{kPa}$ ) hyaluronic acid hydrogel in comparison with the culture on stiffer ( $4.5 \mathrm{kPa})$ gel. FAK was again observed to mediate the stiffness-dormancy response and FAK inhibition increased the survival of dormant cells on stiff hydrogels. More model systems that recapitulate a variety of metastasis sites, both mechanically and chemically, are required to further study dormancy and ultimately prevent metastasis from occurring.

\section{Substrate Surface Patterns}

Invasion and metastasis of cancer cells are likely dependent on abnormal adhesion to the surrounding environment. A better understanding of cell-ECM interaction is required as current prognostic methods do not completely predict metastasis. Patterning cells via controlling cell confinement within predetermined size, shape, and interconnectivity enables testing of cell morphology dependent fate decision, cytoskeletal tension, cell-cell interaction, etc. (Poudel et al., 2012). Also, when cells are seeded on anisotropic or isotropic topography patterns, cells display contact guided orientation to the anisotropic direction or functional behavior changes depending on the scale of the isotropic topography (Lim and Donahue, 2007). Cells may be characterized based on adhesion properties to surface patterned ECM proteins. Specifically, adhesion-based biomarkers in breast cancer cells can be identified by subjecting cells simultaneously to two ECM protein patterned surfaces and pulling the surfaces apart (Figure 2) (Kittur et al., 2017). It was observed that malignant MDA-MB-231 and the organtropic variants TGL/1833, TGL/4175, and TGL/Brm-2a (which are bone, lung, and brain metastatic, respectively) tend to transfer away from basement membrane proteins to collagen type I, while benign human mammary epithelial cells (hMECs) did not show such a transfer. If integrins $\alpha 2$ and $\beta 1$ were inhibited, the transfer to the secondary ECM pattern was blocked. This adhesion profile method may provide crucial data for patient specific treatment depending on cancer cell-ECM interactions. Inspired by this, this section will discuss the control of breast cancer cells with two types of substrate patterning, the creation of topographic patterns in the form of geometric structures that cells may interact with and chemical patterning for selective culturing of cells within defined micropatterned regions. Also, examples using related techniques are included.

Differential response to topographic features is evident between healthy epithelial cells and malignant cancer cells. Malignant MCF-7 cells displayed significantly decreased vascular endothelial growth factor (VEGF) synthesis with lower proliferation and increased apoptosis when seeded on poly(lactic-co-glycolic acid) (PLGA) surfaces with $23 \mathrm{~nm}$ topographic features relative to cultures on smooth or 300 or $400 \mathrm{~nm}$ topographies (Zhang and Webster, 2012). On the other hand, benign epithelial cells had increased proliferation on the $23 \mathrm{~nm}$ features compared to the other surface conditions. This raises the potential of nanoscale topography for anticancer treatment. The anticancer property may be further enhanced with functional surface coating (Zhang and Webster, 2013). Coating the nanoscale features with alginate further decreased the cancer cell function whereas benign cells were unaffected. Beyond cell survival, topographic patterning of microscale features can influence cell migration and cytoskeletal composition. MDA-MB-231 cells had the greatest migration and cell speed on the arc pattern compared with grating pattern or flat control surface (Zhou et al., 2017). The uneven pattern of the arcs could create uneven vinculin focal adhesion protein expression within the cell, possibly causing the increased migration. Taking the topography-induced migration a step further, Chen et al. (2019) biased the direction of cell migration with sawtooth nanoscale ridges formed by multiphoton absorption polymerization. MDA-MB-231 preferentially moved up the sawtooth formations and the M4 metastatic variant of the MCF-10A cell line moved in the opposite direction. With these cases considered, topography could be a powerful tool for affecting breast cancer cell migration and interrogating the molecular motility pathways. 

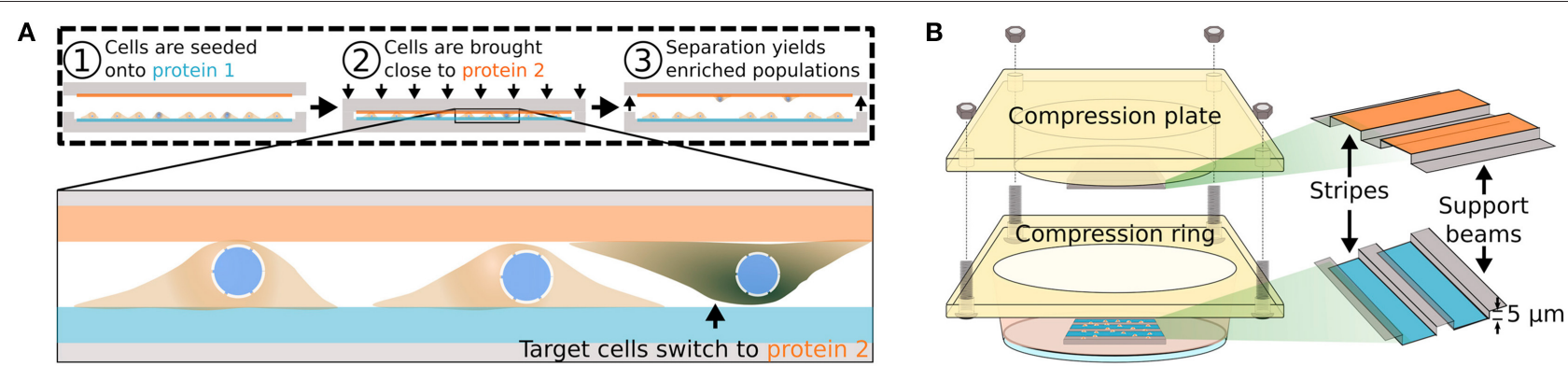

C
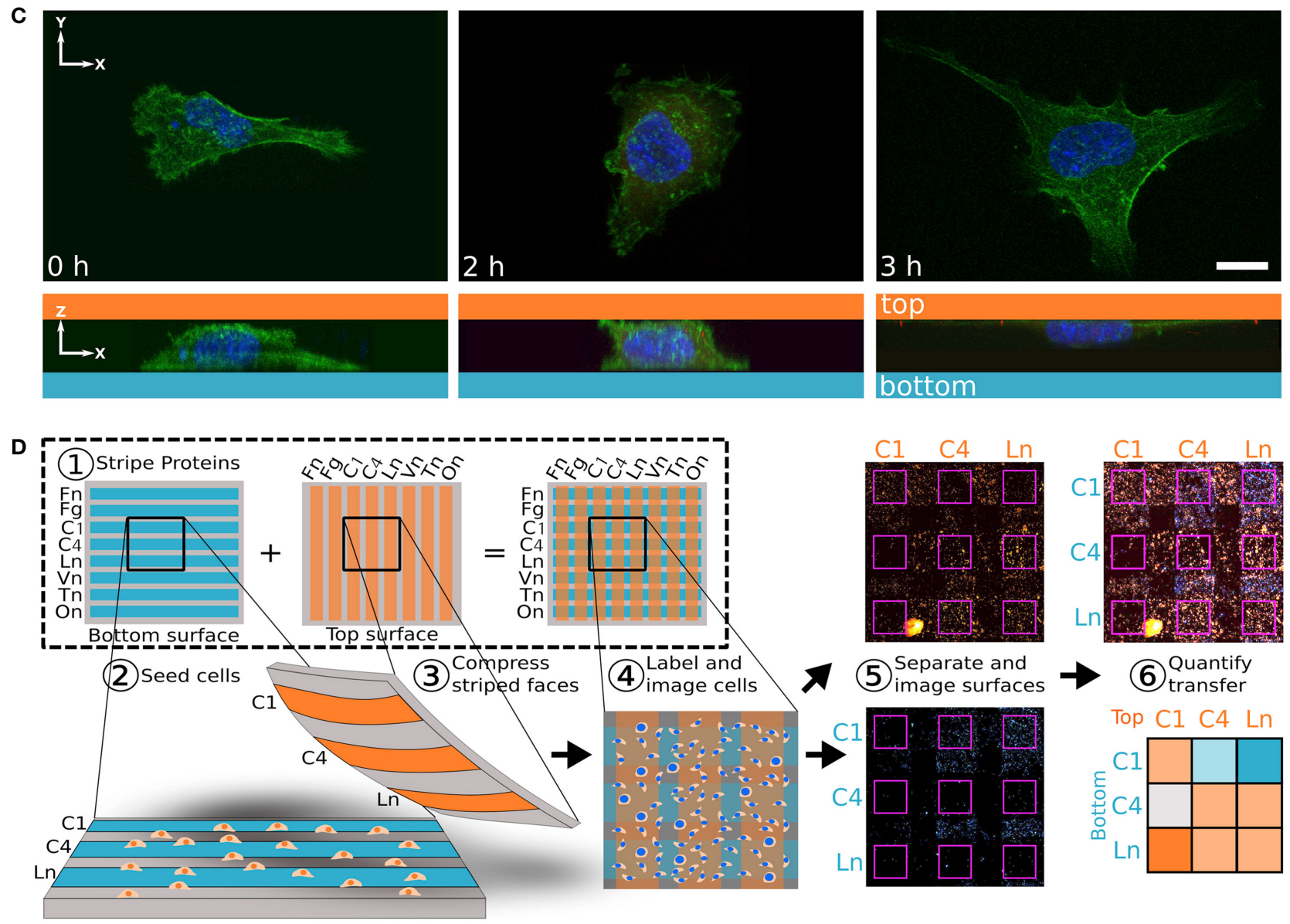

FIGURE 2 | Transhesion assay characterizes cells based on adhesion molecule preference. (A) Schematic for transhesion method. Cells are seeded onto protein 1 and brought into close contact with protein 2. (B) This method is adapted to use a standard petri dish. (C) Cell imaging during transhesion demonstrates cell transfer from protein 1 (left) to protein 2 (right). (D) Eight orthogonal strips of proteins allows for testing of 64 protein combinations at once. At far right is a heat map with darker orange indicating preference for top surface and darker blue indicating preference for original bottom surface. Fn, fibronectin; Fg, fibrinogen; C1, collagen 1; C4, collagen 4; Ln, laminin; Vn, vitronectin; Tn, tenascin C; On, osteopontin. Reprinted with permission from Cell Press (Kittur et al., 2017).

Micropatterning and related techniques can provide advanced methods for developing co-culture and more robust tumor models. In addition to sophisticated lithography-based patterning techniques, making PDMS mold for gradient microfluidic chips could be used to assess cancer cell migration (Zhao et al., 2018). In their study, when cells were patterned on collagen coated plates to measure the migration from the tumor regions into fibroblast regions, MDA-MB-231 had the greatest migration and MCF-7 were relatively stationary. Using defined patterns, cells from tumor regions could later be harvested for the gene expression analysis. Such microfluidic systems were also used to quantify cell migration as affected by chemotherapy drugs or other treatments (Wang et al., 2012). Moving to 3D culture, microfabrication using stereolithography could encapsulate MDA-MB-231, MCF-7, and MCF-10A in gelatin methacrylate to serve as a tumor model (Peela et al., 2016). Using time 
lapse imaging, cells escaping the 3D tumor-like encapsulation could be quantified and migration tracks compared, in which MDA-MB-231 showed pronounced invasion to the surrounding soft matrix. By adding endothelial cells to the 3D patterning models, the vascular interactions may also be investigated, for instance, breast cancer cells were patterned with endothelial fibroblasts in hyaluronic acid and fibronectin gels for the study of angiogenesis in the tumor model (Dickinson et al., 2012). The $3 \mathrm{D}$ printed nanocomposite matrix can also be used for breast cancer cell metastasis to the distal site such as bone (Zhu et al., 2016). In the study, to provide bone-mimicking niche, hydroxyapatite nanoparticles were suspended in a hydrogel. When grown with MSCs which can differentiate to bone-forming cells and deposit bone matrix, the tumor cells formed spheroids similar to those found when breast cancer metastasizes to bone. Interestingly, the cancerous cells in the biomimicking 3D environment displayed higher drug resistance compared to those grown in a $2 \mathrm{D}$ environment, indicating the need of further investigation of 3D bone-like nanocomposites for tumoroid studies.

Besides creating model systems, substrate patterning methods can also be used to interrogate physical properties of cells and distinguish between benign and malignant cells. For example, cell micropatterning was used to assess cytoskeletal stress and arrangement and infer cell state and malignant status (Figure 3) (Tseng et al., 2011). In their study, benign epithelial MCF-10A cells were patterned on fibronectin micropatterns having crossbow, disc, and pacman shapes, and traction force measurement was combined to reveal cell strains at each location on the pattern. It was observed all transformative tumor characteristics were not entirely involved with increased cell contractility, which result is potentially contradictory to the current general view. Since tumor cells often have modified contractile properties, such a system may be useful for assessing cancer cell progression. Similarly, the deformation of the nucleus may reveal the status of cancer cells. The nucleus is physically connected to cytoskeletons via the linker of nucleoskeleton and cytoskeleton (LINC) complex, e.g., nesprin and SUN, and may have a direct role in environment sensing in many cell types (Bouzid et al., 2019). To test the correlation of LINC and nucleus morphology with metastatic potential, topographically patterned micropillars were used to culture MCF-10A, MCF-7, and MDA-MB-231 cells (Matsumoto et al., 2015). Matching with the trend of highly metastatic cells having lower cytoskeletal tension, more metastatic cells displayed higher measures of nuclear deformation associated with significantly reduced LINC expression. The patterning technique has also been attempted to test cellular tension-related multinucleation which is a cancer-related adaptation. Cancer cell fusion is known to contribute to tumor development with fused cells having higher drug resistance. The metastatic MDA-MB-231 cell line formed multinucleated cells within confined patterns, but the typically stationary MCF-7 cell line did not (Zhu et al., 2019). Notably, multinucleated cells first formed on the edges of patterns in higher tension regions and later formed in the middle of patterned regions, revealing a potential strain-sensitive process for the multinucleation. Together, cell micropatterning could be useful for determining cytoskeletal tension and nuclear properties which regulate tumor development and metastasis.

Additionally, cell micropatterning has been utilized to examine dynamic cancer cell processes including migration. A two-state micropatterning with two large rectangular regions connected by a narrow bridge was developed to determine the heterogeneity in migration for established cancer cell lines (Brückner et al., 2020). The migration of MDA-MB-231 cells seeded on one side of the pattern and crossing the small bridge to the second region was recorded and assessed with statistical analysis to reveal potential subpopulations with distinct mechanical and migration capabilities. In a study by Tse et al. (2012), micropatterned cancer cells produced migration leader cells at sharp corners of the pattern in uncompressed controls. When adding the mechanical signal of compression to these patterns, the cells at the pattern boundary were likely to become leader cells. Again suspecting the role of cellular tension in this process, ROCK or MLCK inhibition blocked actomyosin remodeling and diminished the overall migration but did not stop the formation of leader cells.

Studying migration behavior in a patterned environment with physical constriction found the physical constraints to be an important factor in breast cancer migration speed. MCF-7 cells in narrow channels with high constriction migrated faster than those in wider channels, while benign cells (L929, HEK-293) did not migrate faster in confinement but migrated faster in less confined patterns (Yang et al., 2016). These changes were influenced by altered cell stiffness, e.g., cells in the patterns had elevated stiffness as measured by AFM compared to those growing freely without pattern. This system may thus reveal cell behavior relevant to metastasis in narrow capillaries or microchannels. In combination with the topography control of breast cancer cell migration (Zhou et al., 2017) described above, micropatterning and topographical features could provide tools for mechanotyping dynamic aspects of breast cancer cells such as migration and thus determining their metastatic potential.

\section{D vs. 3D Cultures}

Although 2D models provide crucial data on cancer behavior, distinct oncogenic advantages are clear in even simple 3D cultures. We here highlight a few important implications of 2D and 3D cultures being used for breast cancer cell studies and based on that will further motivate the use of 3D culture systems. For a more detailed discussion of a variety of 3D models of breast cancer cells and tissues, see the review by Clegg et al. (2020) published in the journal of Front Cell Dev Biol.

Growing triple negative MDA-MB-231 in a $3 \mathrm{D} \operatorname{poly}(\varepsilon-$ caprolactone) scaffold that mimics breast tumor induced notable gene activation associated with cancer invasion and motility compared to those grown on conventional 2D surfaces (Figure 4) (Balachander et al., 2015). This includes changes in invasive membrane structures (blebbing, lamellipodia) and genes related to cancer stemness (Notch1, Oct3), inflammation (NK-кB, TNF, IL8), and cell-cell and cell-matrix adhesion (integrin, MAPK, LINC, FAK, etc.). Moreover, preconditioning in a 3D tumor-like environment had lasting effects on cells as observed in the tumorigenicity mouse model, e.g., cells from 
A
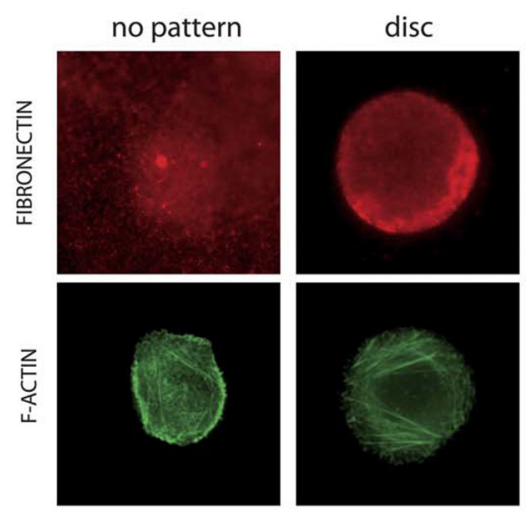

B

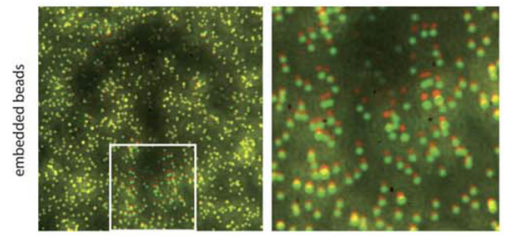

Particle image velocymetry + individual particle tracking

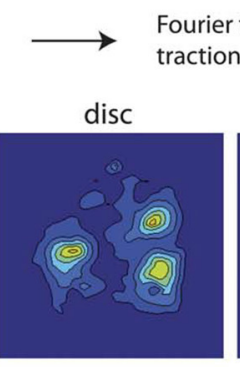

Fourier transform

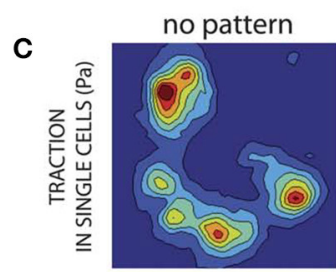

D
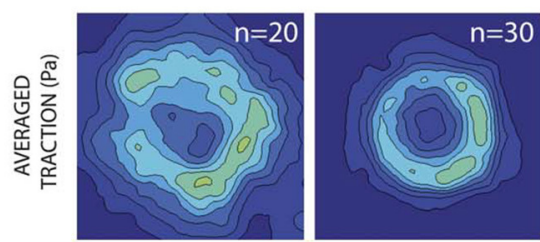

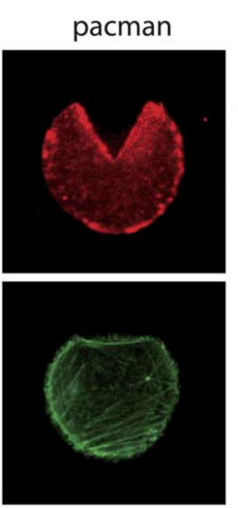

crossbow
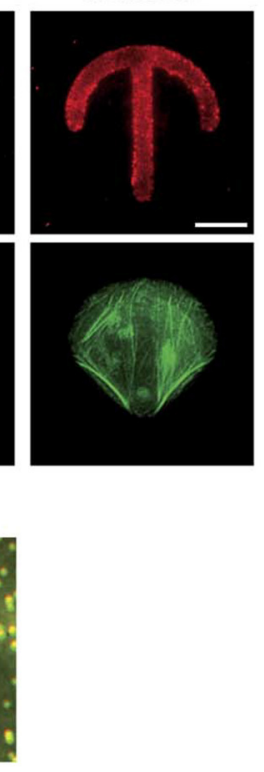

(
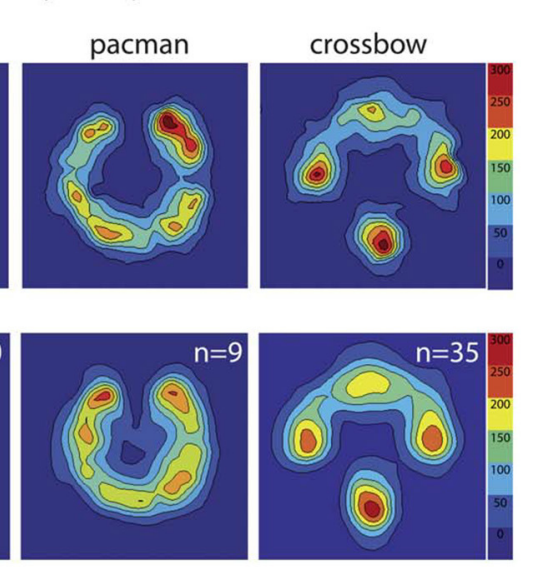

FIGURE 3 | Patterning may be used to investigate cytoskeletal structures and cell traction force profile for mechanotyping. (A) Representative images with staining for F-actin and fibronectin reveal cytoskeletal organization. (B) Beads embedded in the substrate beneath the patterned surfaces can be used to determine displacement fields for traction measurements. Using this method the traction force field can be determined for (C) individual cells and (D) averaged for the pattern. This method provides high resolution data on cell traction forces which may be used to estimate invasive capabilities. Scale bar is $10 \mu \mathrm{m}$. Reprinted with permission from The Royal Society of Chemistry (Tseng et al., 2011).

3D culture developed larger tumors in the mammary fat pad with local invasion and more metastasis to the lungs. Further understanding these processes could lead to better 3D scaffold designs for cancer detection and treatment. Further implementing realistic features to such models, the addition of a basement membrane and surrounding collagen ECM matrix into the 3D model allowed interrogation of the initiation of metastasis and basement membrane breaching (Guzman et al., 2017). In this setup, the basement membrane contained benign MCF-10A cells, while the oncogenically transformed MCF-10A-HRas invaded through the basement membrane endothelial layer and colonized the surrounding collagen matrix. In addition, 3D scaffolds designed to capture metastatic cells could decrease tumor burden at typical metastatic sites and alter the primary tumor milieu (Rao et al., 2016), e.g., poly( $\varepsilon$-caprolactone) scaffolds implanted in mice significantly reduced tumor progression in the liver and brain. Such 3D scaffolds may also serve as a diagnostic tool for monitoring the capture of metastatic cells, and cells captured in this manner may be further analyzed for patient-specific therapy. Adding another dimension of complexity, implanted scaffolds also modified the immune reaction in the primary tumor environment causing an invasion-suppressing profile (Aguado 


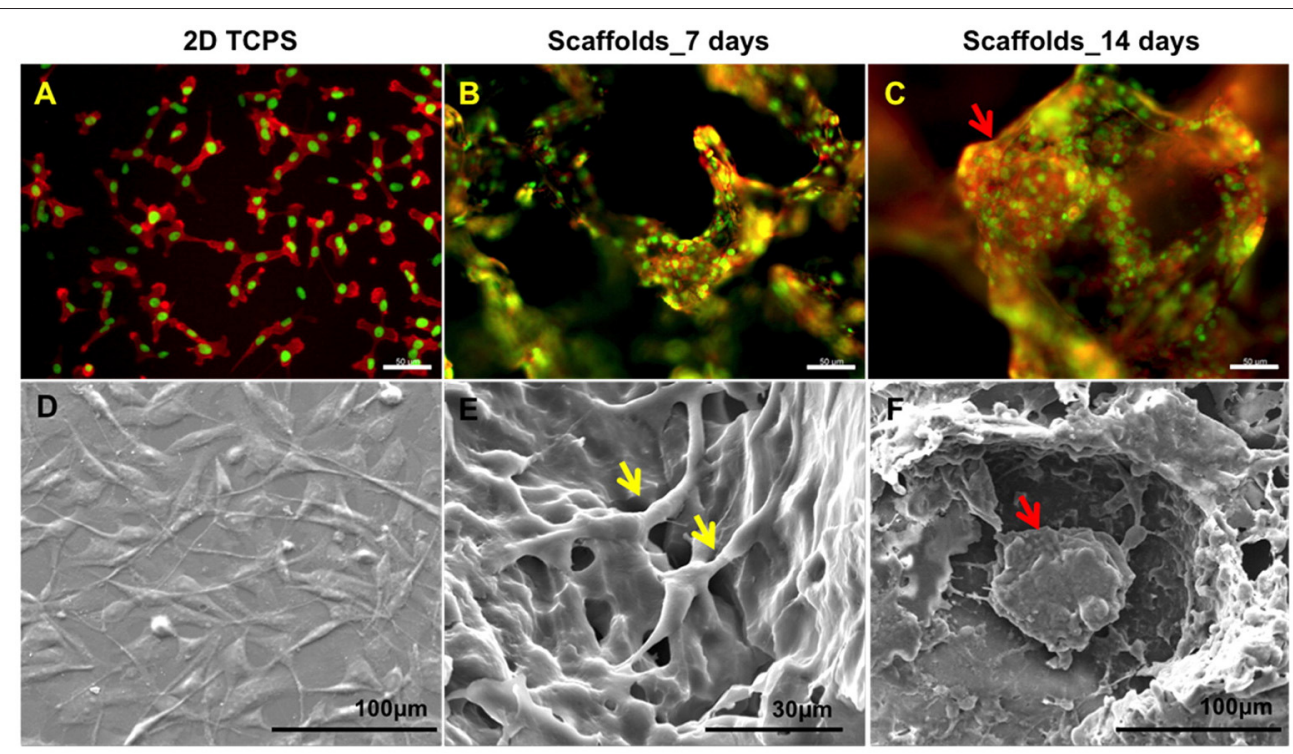

FIGURE 4 | Growing cells in 3D scaffold that mimics breast tumor induced tumor spheroid formation. (A) Fluorescent imaging revealed that MDA-MB-231 on 2D tissue culture plastic (TCPS) failed to mimic the in vivo environment compared to scaffolds at (B) day 7 and (C) day 14. Staining for actin (red) and nucleus (green). Scale bars are $50 \mu \mathrm{m}$. (D-F) are the corresponding SEM images showing detailed cell interaction and tumor formation. Intercellular contacts are noted by yellow arrows and tumor spheroid are noted by red arrows. Reprinted with permission from American Chemical Society (Balachander et al., 2015).

et al., 2018). Based on these data, further targeting 3D scaffold design for the control of oncogenesis and potential treatment and monitoring strategies will greatly benefit the field.

Developing 3D model systems and methods to study cancer metastasis will greatly enhance understanding of cancer disease progression, dormancy, and anti-metastatic drugs. To better mimic human bone environment as a breast cancer metastasis site, a tissue engineering approach was utilized to make nanoclaybased bone model (Kar et al., 2019). MSCs grown in a nanoclay matrix for a month could develop a bone-like tissue, and the construct was then seeded with MCF-7 and MDA-MB-231. This enabled investigations of 3D tumor spheroid formation, cellcell and cell-matrix interactions, and metastasis potential. Taking the perspective of tumors as interconnected organ systems, further work is clearly required to develop model tumor systems that provide realistic cell-cell interactions and recapitulate the response of multiple cell types to mechanical signals (Bregenzer et al., 2019). For example, one study recapitulated 3D blood vessels with a parallel epithelial-lined lumen to investigate the epithelial-endothelial migration in breast cancer (Devadas et al., 2019). This is of significance since the endothelial-epithelial interactions are not well-understood yet for 3D environments. Moreover, the $3 \mathrm{D}$ environment is particularly important for endothelial culture since 2D monolayers of endothelial cells may behave differently than 3D lumens as measured by proangiogenic secretory factors (Jiménez-Torres et al., 2019). In comparison, attempting this work in less biomimetic 2D environments has relied on transwell inserts which lack the physiological 3D lumen structures and fluid flow stimulus.

In addition to modifying cellular crosstalk, the mechanical environment further affects cellular processes such as chromosome segregation. It was observed that fewer segregation defects occur in 3D epithelia culture compared to the segregation in 2D culture conditions (Knouse et al., 2018). In another study exploring the interaction of Ras oncogene with environmental stiffness in benign MCF-10A, Ras promoted cell rounding during mitosis even under confinement conditions, which then prevented chromosome segregation errors (Matthews et al., 2020). The forces generated by cells in this case were proposed to be different in $2 \mathrm{D}$ vs. $3 \mathrm{D}$, with cell division in $2 \mathrm{D}$ resulting in selective loss of cell adhesion to the substrate whereas cell division in 3D may generate forces in all directions.

Taking these microenvironment-dependent cell changes one step further, the epigenetic profile could be sensitive to dimensional and stiffness effects. Stiff 2D culture environments may cause loss of tissue function due to changes in chromatin. In a 3D culture model, stiff ECM induced tumorigenic features in mammary epithelial cells with increased lamina-associated chromatin with more accessible chromatin; a soft ECM in the range of the natural in vivo environment caused in vivo like chromatin profiles (Stowers et al., 2019). Tying in topographic patterning, simply imprinting cell membrane shapes in culture surfaces drastically altered apoptosis and susceptibility to breast cancer drug treatment (Shahriyari et al., 2020). Although 2D cultures have been used a lot for breast cancer work, all of these data from 3D culture setups demonstrate that the culture dimensionality together with the mechanical microenvironment likely affect a variety of cancer cellular processes. Further motivating the move to $3 \mathrm{D}$ culture environments, proper functioning of cellular processes in a more physiological way can be achieved which are inhibited in 2D in many cases. Some representative concepts/findings of the microenvironmental (stiffness, patterning, 2D vs. 3D) control of breast cancer cells are shown in Table 1. 


\section{EFFECTS OF MECHANICAL LOADING MILIEUS}

\section{Mechanical Stretch}

Among various mechanical loading situations to which breast cancer cells could be exposed in vivo, mechanical stretch has been relatively less investigated. Recently, response of breast cancer cells to mechanical stretching was suggested as a marker to distinguish between cancerous and benign cells (Yadav et al., 2019). Under cyclic stretching of $1.4 \%$ strain at $0.01 \mathrm{~Hz}$ frequency, cancerous MDA-MB-231 cells displayed in the initial stretching cycle decreased roundness, increased cell length, and actin cytoskeletal rearrangement perpendicular to the axis of stretching; in the later stretch cycles they showed apoptosis potentially due to increased cellular rigidity. These changes by stretch were specific only for MDA-MB-231 but not for noncancerous cells, indicating that mechanical stretch can be utilized for identifying their metastatic potential.

Cytoskeletal integrity and rearrangement are crucial for cancer cell adhesion and invasion processes. It was found that stretched MDA-MB-231 cancerous cells showed many fold increase in invadopodia, the actin-rich protrusion of the plasma membrane associated with the cancer invasiveness (Ansaryan et al., 2019). Further, the activation of aerobic glycolysis and altered membrane voltage, both indicative of cancer progression, were observed in all breast cancer cell lines tested under stretch, indicating that stretch cue may significantly influence even benign epithelial cells to a cancerlike phenotype.

Tensile stretching may activate motility pathways in multiple subtypes of breast cancer. When MCF-7, MDA-MB-231, and $4 \mathrm{~T} 1.2$ cells were subjected to uniaxial cyclic strain for $48 \mathrm{~h}$ at $10 \%$ and $0.3 \mathrm{~Hz}$ or to a constant $10 \%$ static stretch, both oscillatory and static stretches increased cell proliferation and subsequent transmigration for all three breast cancer cell lines compared with unstretched control (Wang et al., 2020). Typically, MCF-7 and 4T1.2 had substantially larger transmigration in the oscillatory stretch condition. The stretch alteration of cancer cell motility can further be assessed with the invasion assay on endothelial layers, which is utilized to classify breast cancer cell response into distinct behavior categories: apoptosis, adherence to endothelial layer, diffusion through the layer, retraction of endothelial cells, and tearing of the endothelial cells (in the order of increasing invasiveness). In assays evaluating the effect of mechanical stretch on such invasion test (Figure 5) (Ansaryan et al., 2019), the most invasive line tested, MDA-MB-231, caused retraction in the control condition but physically damaged the endothelial cells by tearing the cell membranes in the stretched group. Also, benign MCF-10A, which underwent apoptosis in the static control condition, could diffuse through the endothelial layer after stretch. Similarly, non-invasive malignant MCF-7 adhered to the endothelial layer in the static control but caused retraction of the layer after stretch. Thus, all three cell lines, either benign or cancerous, displayed increased invasiveness when exposed to stretch. The invasion and anticancer drug resistance of MCF10A, MCF-7, and MDA-MB-231 were also elevated in response to static stretch at $15 \%$ for $12 \mathrm{~h}$.
This stretch-induced invasive phenotype persists for long periods even when injected into a mouse model. Cyclic stretch conditioned $4 \mathrm{~T} 1.2$ cells transferred to $\mathrm{BALB} / \mathrm{c}$ mice created significantly larger tumors than unstretched cells (Wang et al., 2020). The stretch conditioned cells also modified the local immune environment via exosome signaling which suppressed anti-tumorigenic immune response. Stretch conditioned cells had significant lasting immune modulation and tumor growth after 10 days, demonstrating that stretch conditioning had lasting effects which persisted when transferred in vivo.

To date, mechanical stretch studies on breast cancer have mostly focused on breast cancer progression. As another perspective, Berrueta et al. (2016) demonstrated that local stretching of tissue in vivo can reduce inflammation, fibrosis, and tumor volume. Mice implanted with breast cancer were subjected to $10 \mathrm{~min}$ of stretching from forelimb to tail daily, and this resulted in 52\% smaller tumor volume compared with unstretched control mice. Some evidence of restoration of T-cell adaptive immunity against the tumor was also found with more abundant lymphocytes in the stretch group. Cytokine mediators of inflammation and cytotoxic immunity were upregulated in the stretched group, revealing a potential mechanism for the stretchbased tumor regulation. This study provides further evidence that factors in the tumor environment and surrounding cells react to mechanical signals in varied and nuanced ways, which may then be utilized in cancer treatments (Berrueta et al., 2018). Complex interventions such as stretching of in vivo tissue and model tissues should be continued to better understand and then resolve the mechanisms of cancer suppression and possible strain-based interventions.

\section{Compression}

The nuanced effects of solid stress on tumor cell progression are poorly understood. Solid stress results from tumors growing in confined tissue environments: the cells increasing in number within the tumor experience compression and the surrounding tissue is remodeled with changes in blood and lymph vessels (Stylianopoulos et al., 2013). Pathological remodeling is also found in the ECM, as is related to ECM stiffening via collagen densification in compressive environments. This in turn affects viscoelastic relaxation, plastic deformation, and interstitial flows in the compressive tumor microenvironment (Ferruzzi et al., 2019). As tumors develop, its interior becomes hypo-vascular and continued cell proliferation causes vascular compression in the periphery. This tends to reduce the efficiency of drug delivery, thus the strategies to alleviate internal tumor stress and reduce cell proliferation may restore efficient drug delivery to the tumor interior (Mpekris et al., 2015). Stress resultant changes in hydrostatic pressure, interstitial flow, and chemical environment all interact to drive or inhibit the cancer progression. In a $3 \mathrm{D}$ MDA-MB-231 hydrostatic model (Tien et al., 2012), pressure on one side of an aggregate inhibited tumor outgrowth from the opposite side. When no pressure gradient was applied, the cells invaded the surrounding collagen matrix, extending tens of micrometers from the original tumoroid. The pressure caused interstitial flow in the aggregate which altered the local chemical microenvironment and resulted in inhibited cell outgrowth. This 
A

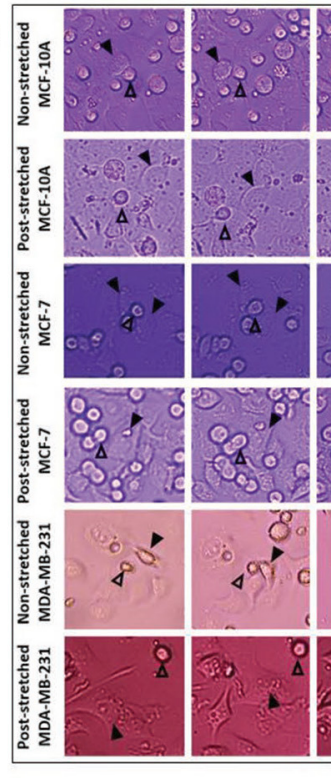

B
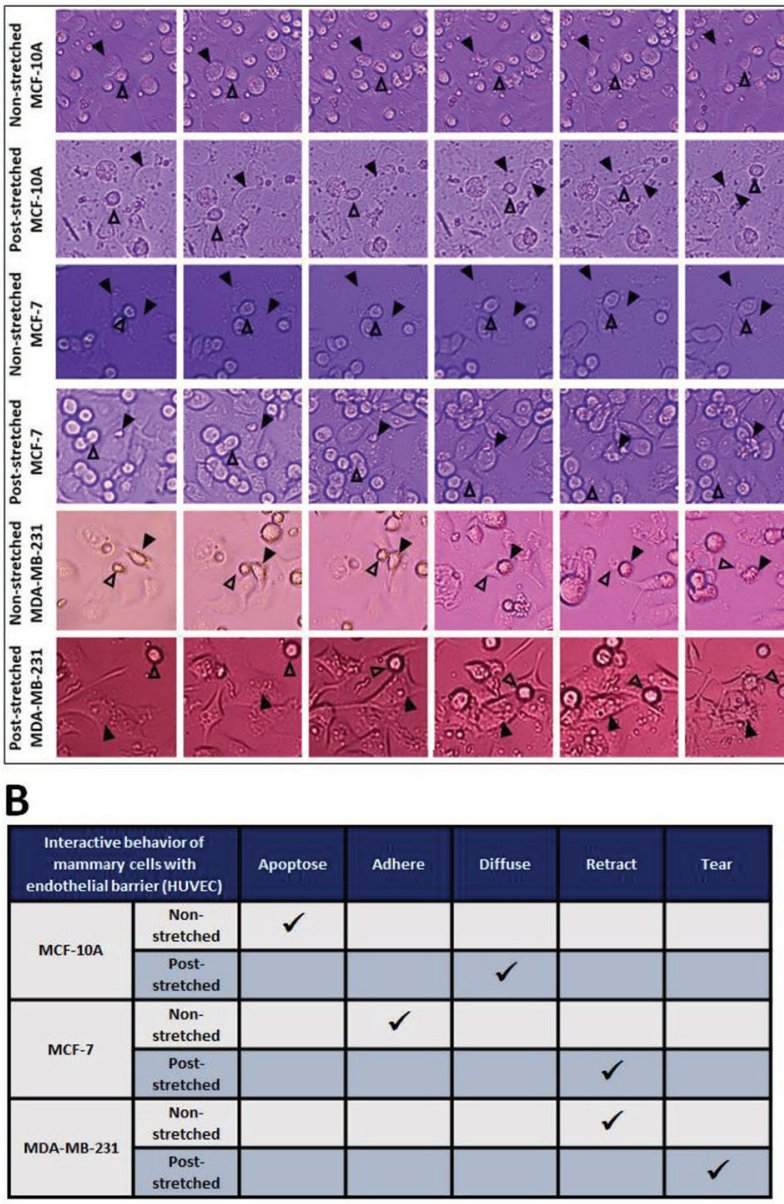
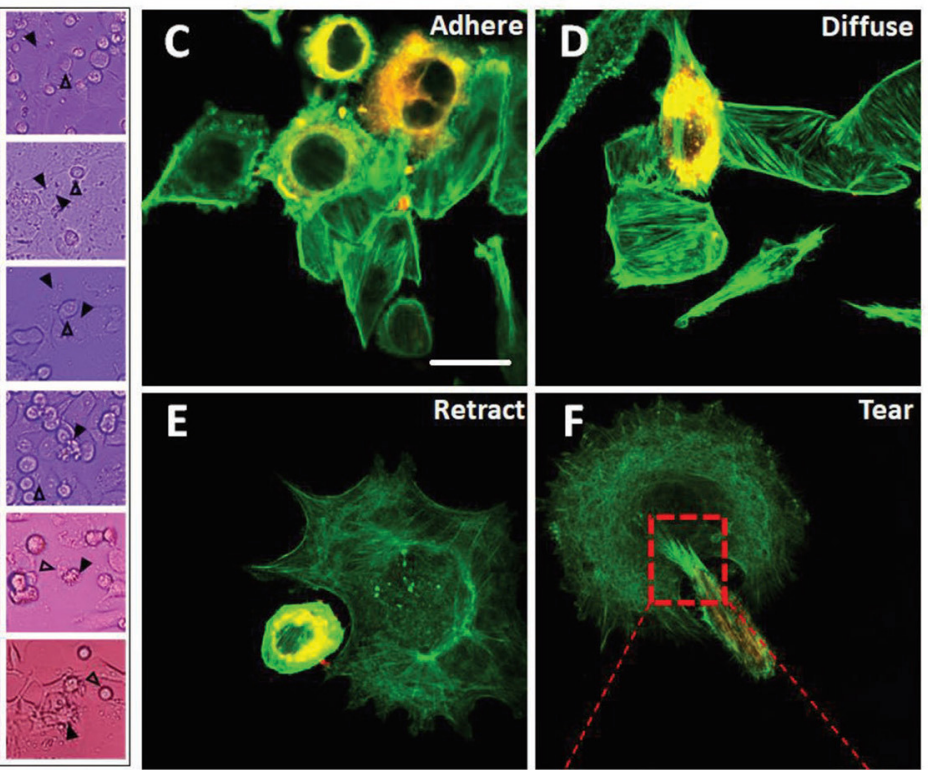

\section{G}

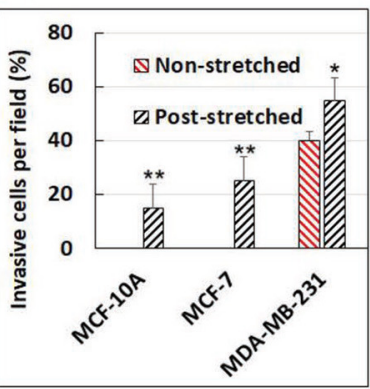

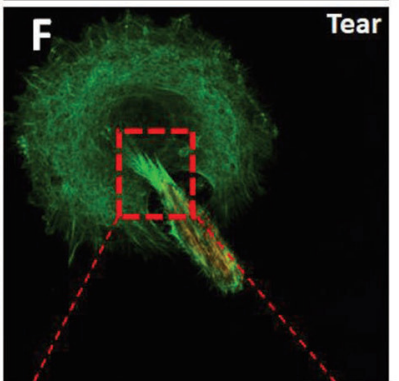

H

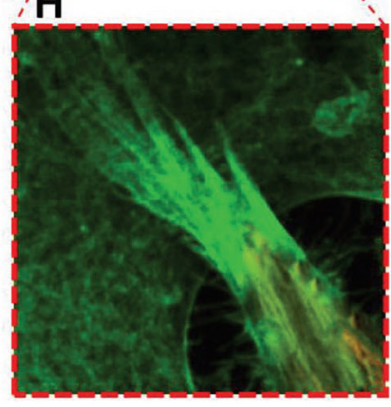

FIGURE 5 | Tensile stretch caused escalation of invasiveness for benign MCF-10A and malignant MCF-7 and MDA-MB-231. (A) Timelapse imaging was used to track cell interaction behaviors over time. Behaviors of interest are marked with arrows. (B) This table summarizes the behavior of the cell lines in the static unstretched group and after stretch. Representative images of (C) adherence, (D) diffusion, (E) retraction, and (F) tearing invasive behaviors. Actin is stained in green for breast and endothelial cells, cancer cells also stained with Dil. Scale bar is $25 \mu \mathrm{m}$. (G) The number of invasive cells per field greatly increased with stretch. ${ }^{\star} p<0.05,{ }^{* *} p<0.01$. (H) Dense invadopodia tore the endothelial cells. Reprinted with permission from Wiley (Ansaryan et al., 2019).

in particular underlines how mechanical stress signals, the local ECM environment, and chemical factors can be interconnected in tumor outgrowth.

Compressive stress can alter cell adhesion to increase adhesive properties and promote invasive phenotypes. For example, in a test using $5.8 \mathrm{mmHg}$ compressive pressure, compressed cancer cells exhibited actin and microtubule realignment with the cell sheets producing distinct leader cells at the periphery to guide the migration activity, and thus displayed persistent and directed migration motion compared with non-compressed control (Figure 6) (Tse et al., 2012). Such changes were only observed for invasive 67NR cells but not for benign epithelial MCF-10A or non-invasive MCF-7. In another study, MDA-MB468 compressed by $15 \mathrm{mmHg}$ pressure increased adhesion by $25 \%$ (Downey et al., 2006). Also, compression of MDA-MB-231 at $50 \%$ strain $(0.05-0.25 \mathrm{kPa})$ for $3 \mathrm{~h}$ in agarose gels increased cell-ECM adhesion and migration while downregulating genes involved in ECM degradation (Demou, 2010). As a potential mechanism, compression of MDA-MB-231 was shown to induce the overexpression of integrin genes with increased expression of PTEN that is found at the leading edge of cell migration. In addition, platelet endothelial cell adhesion molecule-1 (PECAM-1) was downregulated and CD44, typically associated with the central nervous system, was significantly upregulated, revealing potential priming for specific metastasis sites such as brain. Combined, compression could transition tumor cells to a more invasive phenotype as measured by migration and gene expression with potential priming for specific distal metastasis sites.

Besides compression from increased tumor solid stress, invasion through ECM, intravasation, and extravasation also induce unique compressive strains via constrictions on migrating cells. In a study testing MCF-10A, MDA-MB-436, and MDAMB-231 cell migration through narrow constriction using microfluidics and combined chemotaxing with epidermal growth factor (EGF) gradient, counterintuitively, it was evidenced even 
A
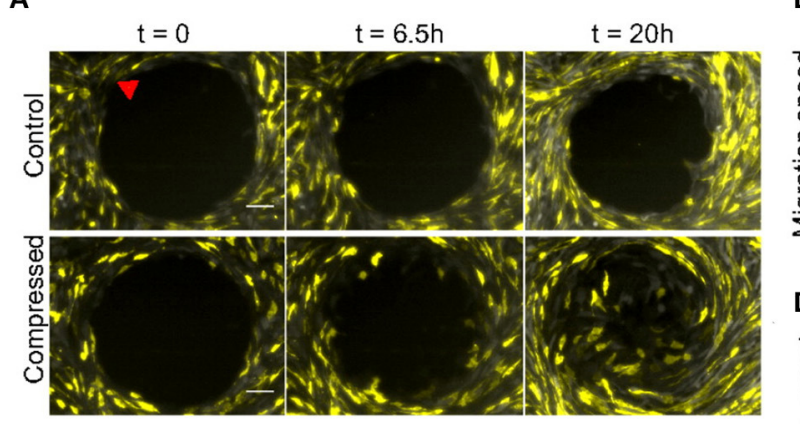

C

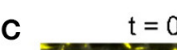

$t=0 \quad t=6.5 h$
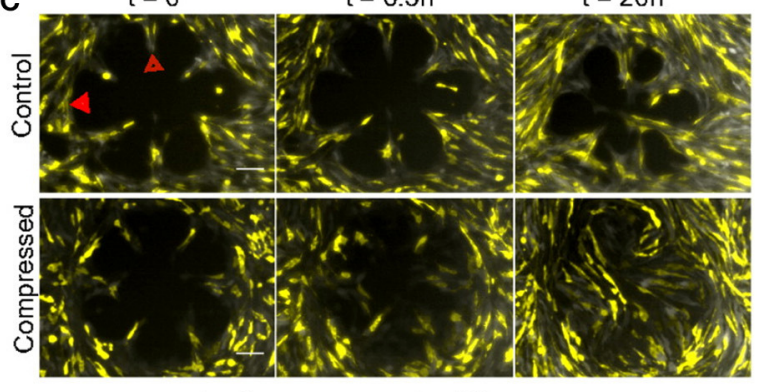

F

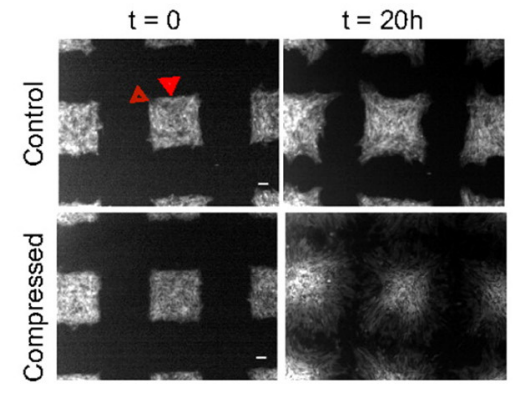

B
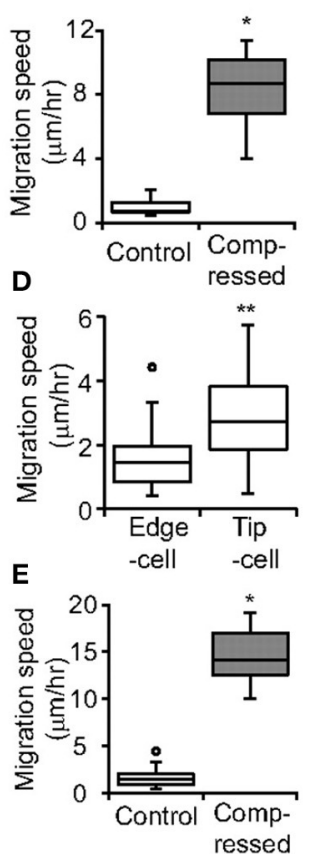

G

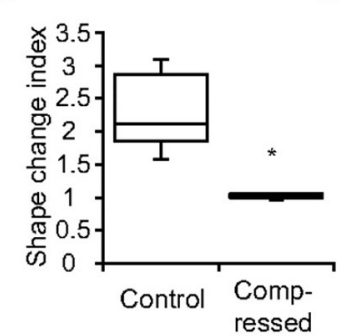

FIGURE 6 | Compression caused enhanced invasion properties and formed more leader cells. In uncompressed controls, the pattern shape (A) circle, (C) rosette, (F) and square controlled leader cell formation. Scale bar is $100 \mu \mathrm{m}$. Compression greatly increased leader cell formation on the patterns with increased (B) speed in the circular pattern. (D) Migration speed of tip cells is greater than edge cells. (E) Migration speed is greater for compressed cells. (G) The square pattern shape change can be assessed with the shape index which significantly changed under compression. ${ }^{*} p<0.005$, ${ }^{\star *} p<0.05$. Solid arrow marks edge cells and hollow arrow marks tip cells. Reprinted with permission from National Academy of Sciences (Tse et al., 2012).

benign MCF-10A cells may have a natural propensity to migrate through constrictions (Ficorella et al., 2019). The mesenchymallike MDA-MB-231 used blebs to pass through the constriction, whereas the MCF-10A primarily used lamellipodia with some blebbing. The less aggressive metastatic MDA-MB-468 did not adapt or optimize for passage through the constriction, resulting in low migration through the passage. These results may need to be interpreted considering the dimensionality and mechanical properties of the local environment. Mesenchymal migration strategies have been observed in 2D migration, while in $3 \mathrm{D}$ environments with constrained channels the cytoskeletal adaptation may reduce the dependence on focal adhesions. It was observed that MDA-MB-231 migration was reduced by $\beta 1$ integrin inhibition on 2D substrate but not affected in 3D (Balzer et al., 2012). The directed migration in 3D confined environments relied more on microtubule polymerization but less on actin polymerization as is dominant in $2 \mathrm{D}$. Understanding migration strategies in compressive constriction situations may be key to developing anti-metastatic treatments to block invasion through the ECM and intravasation.

How compression contributes to cancer cell survival and progression is largely unknown. Compression may contribute to tumor cell survival in the hypoxic conditions by activating glycolysis genes and adapting cell metabolism and microRNA (miRNA). It was observed that metabolic, EMT-related, and angiogenesis genes were all upregulated in compressed patientderived cancer-associated fibroblasts compared to the static control (Kim et al., 2019). In vitro compression models also found upregulated glycolysis genes in MDA-MB-231 and migration-related genes in SK-BR-3 cells. In a study using a $3 \mathrm{D}$ agarose scaffold-alginate bead model, compression enhanced tumor phenotype in MDA-MB-231 and BT-474 cells and VEGF expression in the breast cancer cells and associated fibroblasts (Kim et al., 2017). This may act through the compression regulation of miR-9 which can regulate lamin and integrin and is known to be downregulated in advanced breast cancer. 


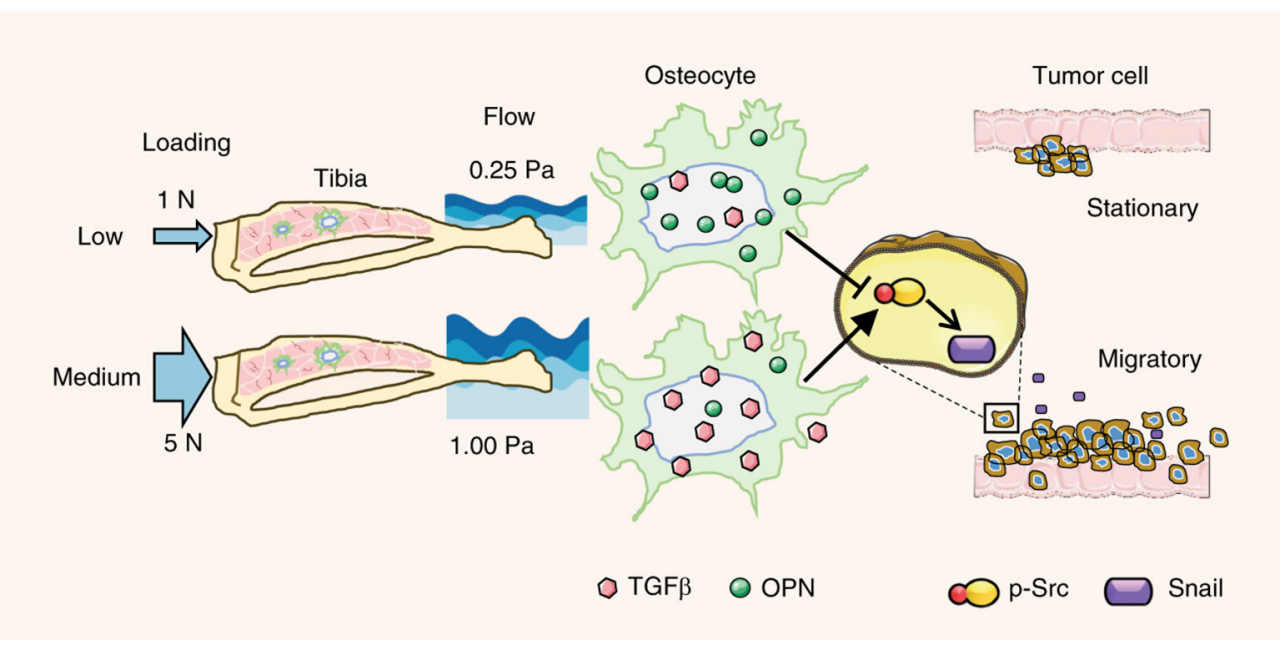

FIGURE 7 | Loading magnitude regulates tumor destruction of bone via osteocyte signaling. Low levels of compressive loading of bone in vivo and of osteocyte shear in vitro caused suppression of cancer migration. Higher compressive loading of bone and shear of osteocytes caused activation of breast cancer migration by upregulating Src and snail. Adapted with permission from Springer Nature (Fan et al., 2020).

The expression of miR-9 was decreased in MDA-MB-231 and BT-474 breast cancer cells and cancer-associated fibroblasts but not in low metastatic MCF-7 or SK-BR-3, highlighting potential differences in miRNA among breast cancer subtypes. In another study, MDA-MB-231 cells had the largest response to compression which downregulated miRNAs associated with tumor suppression genes such as apoptosis, adhesion, and cycle arrest, whereas MCF-7 and BT-474 cells had lower response (Kim et al., 2016). Such interrogation may reveal miRNA targets for disrupting breast cancer and cancer associated fibroblast communication via paracrine signaling. Together, compression could regulate miRNA expression critical for tumor development and suppression, and therapeutics based on inhibition of tumor miRNA or enhancement of suppressive miRNA would provide targeted cancer treatments.

Inducing necrosis over apoptosis in cancer cells encourages immune cell recruitment. In addition to above-mentioned effects, compression was also tested for tumor cell necrosis. It was observed that necrosis was induced in BT-474 and MDA-MB-231 by compression in a force and time-dependent manner (Takao et al., 2019). Dynamic compression in the range of about 10 $\mathrm{kPa}$ could induce cell death dominated by necrosis; dynamic compression to cause this effect was with less magnitude than that required in static compression.

Similar to the initial tumor progression, the mechanical forces in distal metastasis sites can also guide cancer cell behavior. At the primary tumor site, cancer-associated fibroblasts contribute to tumor response and release cytokines upon stimulation. Fibroblasts interact with both the chemical and mechanical environments, providing additional mechanical cues and filtering of mechanical signals to the tumor (Bregenzer et al., 2019). In distal metastasis sites, this role is filled by other cells which interact with the metastasized cells. In one study, compressive loading of the bone in the mice that received intratibial MDAMB-231 injection inhibited the tumor growth and prevented osteolysis associated with tumors (Lynch et al., 2013). This effect may act through the decrease of Runx 2 in the loading group which is expressed by tumor cells and has an effect in both osteoclast and osteoblast regulation. In the absence of loading, the tumors continued to grow and osteolytic activity was increased producing a completely degraded tibia. Regulation of tumor development by compression may depend on the loading magnitude. For the tibia loading applied to mice injected with EO771 and 4T1.2 tumor cells, loading with $1 \mathrm{~N}$ reduced or prevented bone destruction from tumor activity, while $5 \mathrm{~N}$ induced osteolysis with significant bone loss and microcracks (Figure 7) (Fan et al., 2020). In parallel, if osteocyte-derived conditioned medium and fluid flow-treated medium were given to breast cancer cells at 0.25 or $1 \mathrm{~Pa}$ shear stress, both media under lower shear stress resulted in mesenchymal-to-epithelial transition (MET) while flow-conditioned medium at high shear triggered epithelial-to-mesenchymal transition (EMT).

\section{Fluid Shear}

Study of cell migration directed by fluid flow-induced shear is of significance for understanding how cells migrate out of tumors with the interstitial flow environment and for understanding how cells respond in active distal migration environments in the vascular, lymph, and metastatic sites. The direction of tumor cell migration is dependent on environmental factors including dimensionality, matrix material, cell density, flow velocity, and cell receptor activity, and MDA-MB-231 cells showed heterogeneous responses to microfluidic flows depending on the environmental parameters (Polacheck et al., 2011). In another study, fluid shear as a whole increased breast cancer cell motility in a $3 \mathrm{D}$ environment but again did so in a heterogeneous manner, so that simple averages of cell behavior might not reveal an accurate picture of migration (Haessler et al., 2012). Targeting higher shear flows as may be found in vessels, our group demonstrated that cells with higher metastatic potential 


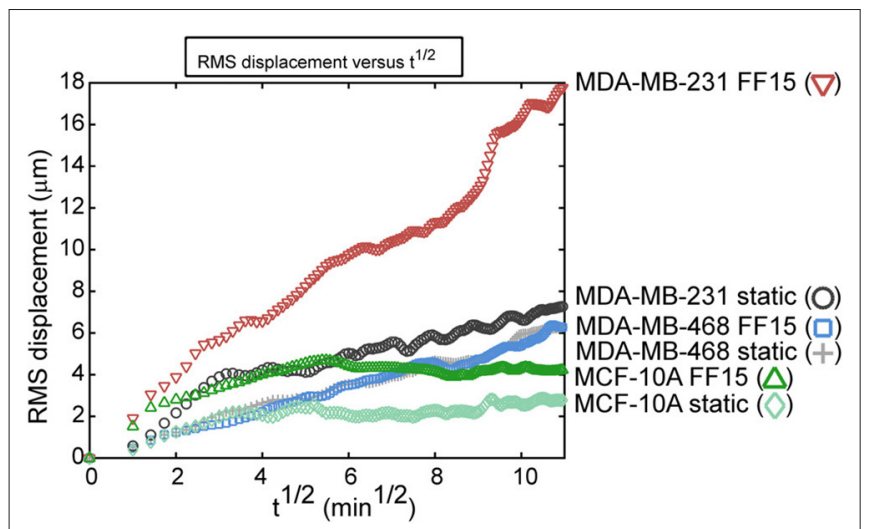

FIGURE 8 | Group migration trends as assessed by root mean square (RMS) displacement reveal that intrinsic metastatic potential and fluid shear activation combine to increase breast cancer cell migration. Benign MCF-10A cells had low migration capabilities in both the $15 \mathrm{dyne} / \mathrm{cm}^{2}$ fluid flow (FF15) and static group. The less aggressive MDA-MB-468 cell line had almost no effect from fluid shear stimulation. Contrasting this, the aggressive MDA-MB-231 had higher migration in the static group and the migration capability was greatly increased by fluid shear stimulation. Reprinted with permission from American Society of Mechanical Engineers (Riehl et al., 2020).

display greater sensitivity in migration to fluid shear (as assessed by root mean square, RMS, displacement of all participating cell migrations as a holistic measure, Figure 8) (Riehl et al., 2020). With a parallel plate flow chamber producing $15 \mathrm{dyne} / \mathrm{cm}^{2}$ shear stress, we observed that flow caused highly metastatic MDA-MB-231 cell migration along the flow direction with higher displacement, greater speed, and less pausing. In contrast, less metastatic MDA-MB-468 was less responsive to flow and benign MCF-10A had the lowest migration potential under shear. Combined, these studies highlight how cells may have heterogeneous responses in migration under flow, which requires investigation in a range of flow environments simulating tumor or distal metastasis sites and for various breast cancer cell types.

In terms of potential mechanistic sensors to regulate migration under flow, for MDA-MB-231, interstitial flowinduced directional migration was blocked when CCR7 was inhibited (Polacheck et al., 2011). Also, FAK was activated specifically in cells that migrated against the flow direction and blocking Src kinase reduced migration against the flow. In another study targeting interstitial flow as present in tumors, it was found cells were polarized for migration through $\beta 1$ integrin (Polacheck et al., 2014). Cell adaptation via paxillin focal adhesion anchor protein also had a key role in this process, producing biased migration in the flow direction, e.g., when paxillin was inhibited, MDA-MB-231 cells no longer migrated against the flow and instead moved with the flow. The details of integrin and related focal adhesion protein regulation under flow are still undetermined. It was recently proposed as part of the remodeling and adaptation response, integrin and focal adhesion components are trafficked by endocytosis (Tang et al., 2020). Fluid shear stress activated focal adhesion turnover in MDA-MB231 by endocytosis, and $\beta 1$ integrin was internalized and recycled back onto the cell surface during migration. This process was observed to be increased by shear flow stimulation.

Microfluidic platforms can provide an easily customizable solution for simulating tumor environments, for example, the microfluidic parallel lumen device targeting endothelial and epithelial co-culture as described above (Devadas et al., 2019). Devices may further be customized for each segment of the metastatic process. In a study by Chen et al. (2013), a 3D microvascular network was grown in a microfluidic platform to study cancer cell extravasation across endothelial cells and out of the lumen under shear stresses of $0.012-0.48 \mathrm{~Pa}$ relevant to those in venular microvessels. The transmigration rates were correlated with known metastatic potential with MDA-MB-231 having a rate of $13 \%$ and MCF-10A with only $5 \%$. Revealing the mechanical aspect of transmigration, cells that were mechanically trapped or constricted had a transmigration rate of $48 \%$ compared to $10 \%$ for non-constricted cells. Understanding how flow in the blood, cell-cell interactions, and constrictions work together to influence the metastatic cascade could reveal new treatment targets. Cognart et al. (2020) tested with microfluidics epithelial-like and mesenchymal-like cells in their responses to the constriction in a flow. Constrictions in the flow could reveal the viscoelastic behavior of the cells tested: MDAMB-231 demonstrated more plastic behavior evident in faster times for passing through subsequent constrictions after the first constriction, while this memory was less pronounced in epithelial-like SK-BR-3 cells. This work demonstrated changes in cancer cells generated by the mechanical stimuli that arise from circulatory conditions including constriction.

Flow causes a multitude of changes in cancer cell survival closely related to EMT including the development of stem celllike properties. Although considered to be distinct, both EMT and stemness equip cells to migrate and survive and may combine to create migrating cancer stem cells. The flow shear may promote the EMT process and render cancer cells to be more aggressive by activating embryonic-like stem properties through the deactivation of extracellular signal-regulated kinase (ERK) and GSK3 $\beta$ (Choi et al., 2019). In a 3D flow bioreactor, shear stress significantly increased the area of MDA-MB-231, MDAMB-468, and MCF-7 cells while decreasing roundness, which may be associated with higher propensity to metastasize (Novak et al., 2019). Tumor cells in the blood vessels, or circulating tumor cells (CTCs), are in a suspension state exposed to blood flows. It was found that cell suspension significantly increased the adhesion ability of MDA-MB-231 to the endothelium via forming stress fibers and focal adhesions with $\beta 1$ integrin (Zhang and Lv, 2017). When suspended cells are injected to a mouse model, they showed significant increase in the metastasis to the lungs with more than double the mass in the lungs compared with cells that were grown in an adhesive environment (Figure 9) (Zhang et al., 2018). The survival of the suspended cancer cells was found to decrease with increasing shear level over time for MDA-MB-231, MDA-MB-468, and MCF-7 cells (Xin et al., 2019). Cells that survive these conditions were proposed to be responsible for generating metastatic tumors. These cells had unique morphology when replated, displayed lower stiffness, and were proposed to be a hybrid of epithelial/mesenchymal 


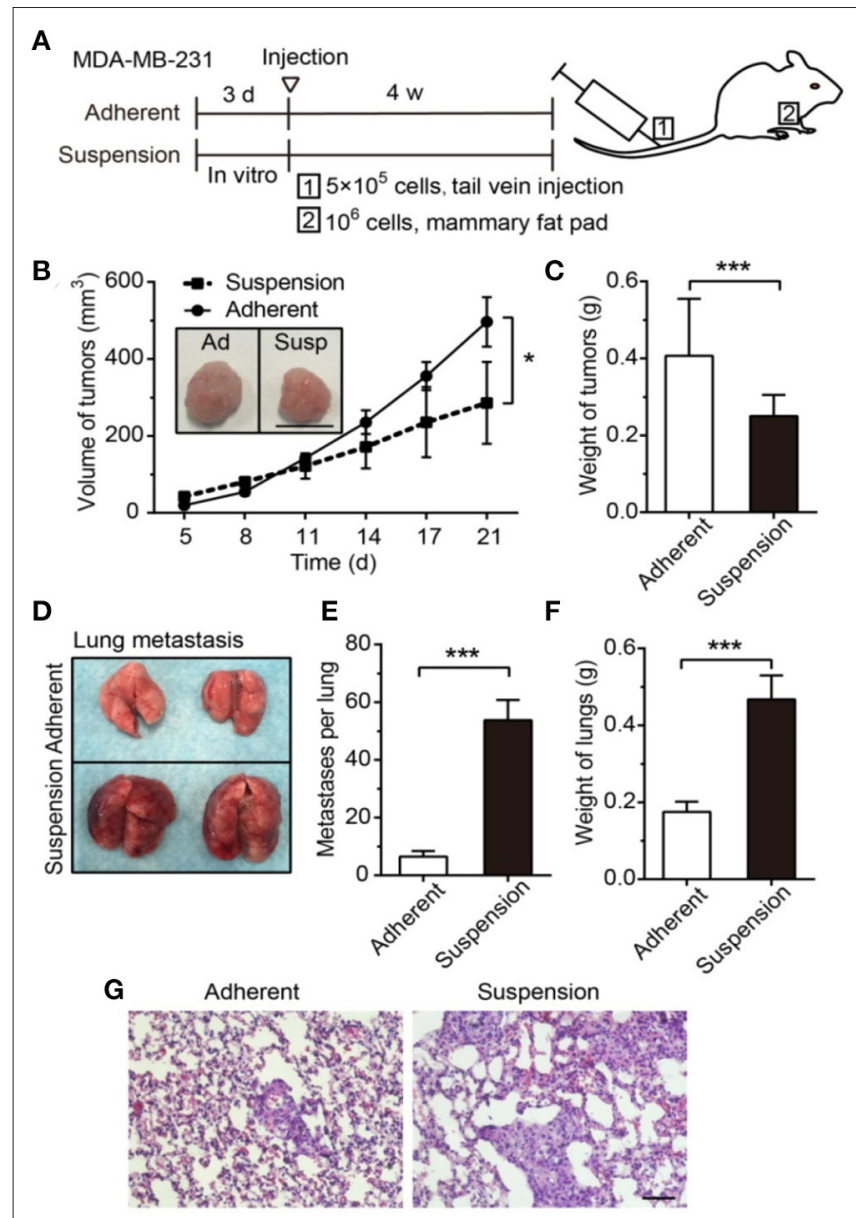

FIGURE 9 | Suspension of cancer cells, as may be experienced in vascular migration, decreased volume of primary tumor but greatly enhanced lung metastasis. (A) The experimental timeline for 1: metastasis study for cell injected in the tail vein and 2: primary tumor from cells injected to the mammary fat pad. Suspension decreased the primary tumor (B) volume and (C) mass. Scale bar is $1 \mathrm{~cm}$. (D) Representative lung images after 28 days reveal that suspension of cancer cells greatly increased metastasis as evident in the (E) number of metastasis and $\mathbf{( F )}$ mass. (G) Larger regions of metastasized cells in the suspension group is evident in $\mathrm{H}$-E staining of the lungs. Scale bar is $50 \mu \mathrm{m} .{ }^{*} p<0.05,{ }^{* \star *} p<0.001$ Reprinted with permission from Ivyspring International Publisher (Zhang et al., 2018).

phenotype. Inhibiting actomyosin blocked flow-induced cell death, whereas activating actomyosin decreased cell survival. Moreover, cells with altered mechanical properties that survived shear in suspension were chemoresistant to the chemotherapy drug, fluorouracil, suggesting a connection between drug resistance and flow mechanotransduction. With more evidences that show flow shear may result in chemoresistance, e.g., to paclitaxel (Novak et al., 2019) and doxorubicin (Triantafillu et al., 2019), more holistic testing may be required to add mechanical signals such as flow to the current $2 \mathrm{D}$ monolayer high throughput screening test.

Flow mechanotransduction is altered in cancer cells and potentially also regulates cell metabolism. Fluid flow in 3D interstitial flow environment caused increased activity of AMPactivated protein kinase (AMPK), with MDA-MB-231 being more sensitive than MCF-10A (Steele et al., 2019). AMPK is recognized as a tumor suppressor and is activated when cell energy is low as based on ratios of ATP and other metabolic molecules. The AMPK activation data in cancer have led to the hypothesis that the AMPK effect can be location-dependent, e.g., flow caused AMPK activation localized to mitochondria and inhibiting FAK and Src removed this effect. Further providing evidence on the location dependent AMPK response in MDAMB-231 to fluid shear was reported including plasma membrane, cytosol, nucleus, mitochondria, and Golgi apparatus (Guo et al., 2020). It was also observed that myosin II, a contractile protein, was required for mitochondrial AMPK mechanotransduction under flow and fluid shear-induced cell migration.

The research has largely concentrated on tumor cells and their response to mechanical signals. For a more holistic measure, the role of surrounding cells and other cell phenotype transitions may need to be considered. Cancer associated fibroblasts may be generated through the endothelial to mesenchymal transition (EndMT). Human umbilical vein endothelial cells (HUVEC) underwent EndMT dependent on the stiffness of 3D collagen matrix and glycosaminoglycan (GAG) concentration, which process was accelerated by low levels of shear flow (Mina et al., 2017). GAGs are important components of glycocalyx shear sensing which may be activated in both endothelial cells and cancer (Tarbell and Cancel, 2016). It was shown that shear stress activated GAG cell surface receptors and GAG synthesis while decreasing breast cancer spheroid size. As a result, shear stress significantly activated the interaction of endothelial and breast cancer cells, thus increasing cancer cell migration rate, traveled distance, and proliferation. In this model, the mechanically active tumor environment activated endothelial EndMT, which further promoted cancer cell growth and metastasis.

Endothelial cell surface receptors can also be activated by the shear flow environment. Such activation enables endothelial remodeling and also may be used by cancer cells for attachment and migration. Shear flow in the range of that experienced in a medium-sized vessel increased the attachment of MDA-MB-231 to HUVEC (Gomes et al., 2003). Simulating the inflammation in a tumor environment, TNF- $\alpha$ treatment of a microvessel network also caused HUVEC layer permeability resulting in a 2.3-fold increase of cancer transmigration rate (Chen et al., 2013). In addition to factors like TNF- $\alpha$, migrating cancer cells in vasculature can interact with E-selectin on endothelial cell surface and soluble E-selectin shed in shear environments (Kang et al., 2016). Soluble E-selectin promoted the migration and adhesion under fluid shear for MDA-MB-231 and MDA-MB468 cells both of which are CD44+/high . In contrast, CD44-/low cells, MCF-7 and T-47D, had negligible response to shear related adhesion in the presence of soluble E-selectin. Enhancement of CD44 ${ }^{+/ \text {high }}$ adhesion activated by E-selectin was abolished with FAK inhibition. Furthermore, adhesion of MDA-MB-231 treated with E-selectin caused permeabilization of the endothelium which could aid migration out of the vasculature. These effects were demonstrated in a mouse model when tumor cell homing to lungs was increased 2.5 -fold when treated with E-selectin. To 
prevent such a process from occurring, an E-selectin targeted aptamer (ESTA) may be developed. An ESTA was found to inhibit shear related adhesion of MDA-MB-231 and MDA-MB-468 to endothelial cells, and the results were confirmed with in vivo ESTA injection resulting in a $92 \%$ reduction in metastasis of $4 \mathrm{~T} 1$ breast cancer cells (Kang et al., 2015). A continued study of endothelial activation, receptor profiles, and fluid shear response will be required to understand multiple aspects of the metastatic cascade. Table 2 shows some representative concepts/findings of the mechanical loading milieus (stretch, compression, fluid shear) control of breast cancer cells.

\section{POTENTIAL MECHANOTRANSDUCTION MECHANISMS}

Studies on environmental cues and mechanical loading signals have proposed mechanotransduction pathways involved in numerous aspects of the metastatic cascades of breast cancer cells. Identified pathways will be useful for suggesting treatment targets and also be used as diagnostic markers. Mechanotransduction elements and pathways have been described throughout this review. Some of the key pathways of breast cancer cell progression will be further highlighted in this section.

Starting at the cell periphery, plasma membrane proteins, glycocalyx or pericellular matrix, cell-ECM adhesion, and cell-cell junctions all have roles in cancer adaptations. ECM connections via transmembrane integrins are integrated into the cell at focal adhesions, which have complexes of physical anchoring proteins that can also be involved in mechanosignaling pathways. Elements associated with focal adhesion signaling and downstreams include FAK, vinculin, paxillin, talin, Src, PI3K, ERK, etc. Focal adhesion mechanotransduction is implicated in many processes involving strain sensing as was found in stretch or flow conditions. Each of these elements interacts with numerous pathways and connect with other mechanotransduction subsystems. For example, the focal adhesion protein talin connects integrins to F-actin, allowing force transmission and adaptation throughout the cell. Talin can be overexpressed in breast cancers and blocking talin could inhibit cancer invasion and migration (Wen et al., 2019). The talin-vinculin-actin filament linkage can transduce forces inside the cells and as a result regulate cancer metabolism. It was shown that through this focal adhesion linkage the mechanical signals activate the PI3K/Akt/mTor pathway causing oncogenic adaptation in cell metabolism and apoptosis resistance (Rubashkin et al., 2014). Further highlighting the interconnection of mechanotransduction pathways, PI3K also participates in the Racl-JNK pathway which regulates cell motility and cytoskeletal adaptation (Carey et al., 2017). In addition to the results presented in previous sections including integrins, FAK, etc., clearly, focal adhesion-based mechanotransduction adapts to the mechanical environment, providing direct force transmission routes to other elements and further triggering cell processes involved in cytoskeletal adaptation, migration, and even metabolism and apoptosis.
Moving inwards, the cytoskeletons and associated regulators of cytoskeletal turnover and tension can mediate cell migration and adaptation processes. Of particular interests are Racl and RhoA which have opposing effects and locations in polarized migrating cells. Racl and RhoA are coordinated in breast cancer cell migration, so that Racl causes actin assembly at the leading edge of the cell migration and RhoA regulates cell contractility with more localized at the trailing edge (Byrne et al., 2016). In another view, migration driven by Rac1 has been reported to be mesenchymal in nature, whereas migration dependent on RhoA/ROCK is more amoeboid in nature and suited for migration through less dense matrices such as collagen. Targeting both Rac1 and RhoA/ROCK pathways may therefore be an effective strategy for preventing tumor cell invasion and metastasis (Jones et al., 2017).

Prevention of metastasis to bone targeting RhoA/ROCK pathway has been investigated considering Rho/ROCK has aberrant expression in breast tumors. Inhibiting ROCK decreased cell proliferation and metastasis to bone both in vitro and in vivo mouse model (Liu et al., 2009). Induced overexpression of ROCK in normally non-metastatic MCF-7 resulted in a metastatic phenotype, inducing metastasis to the hindlimbs and liver. The mass of the metastatic tumors was decreased by $77 \%$ with the application of ROCK inhibitor, Y27632. The context and local tissue architecture may need to be considered when interpreting such results, considering environment and dimensionality influence cell behavior and mechanotransduction pathways as described earlier. ROCK was reported to be elevated in T4-2 cancer cells but not in non-malignant S1 cells, but such a difference was only observed in 3D but not in 2D culture (Matsubara and Bissell, 2016). Since ROCK acts through cytoskeletal tension signaling, related cytoskeletal elements can also play a role. Increased myosin light chain (MLC) phosphorylation was found in malignant cells in accordance with unorganized F-actin; ROCK inhibition reorganized F-actin and repolarized the cells. As another element, it was shown that triple negative cells had low responsiveness to ROCK inhibition but this effect was reversed when cells were manipulated to overexpress E-cadherin, proposing a connection among cytoskeleton, ROCK activity, and E-cadherin cell-cell adhesion. Together, forces and mechanotransduction pathways are transmitted from anchorage points to the cytoskeleton, for both cell-matrix junction and cell-cell junction, and vice versa.

Mechanotransduction interfacing with the nucleus includes direct links which transmit forces to the nucleus and a host of mechanotransduction cascades that regulate genes. One route of mechanical information transfer is the translocation of proteins to the nucleus. As discussed above, YAP can translocate to the nucleus in response to the local strain environments, both static and dynamic. YAP and the transcriptional co-activator PDZ-binding motif (TAZ) are transcription factors typically associated with the Hippo pathway. These factors are particularly relevant for cancer research, since these have been implicated in the formation of cancer stem cells and EMT transition in response to mechanical forces (Jabbari et al., 2015). Some catenin subtypes are also able to translocate to the nucleus in response to mechanical signals, as is often coordinated 
with cadherin cell-cell junctions. In stem cells, the canonical $\mathrm{Wnt} / \beta$-catenin pathway is involved in stem cell self-renewal. The canonical and non-canonical Wnt pathways also regulate cancer stem cell development and may provide a number of potential treatment targets for cancer stem cell elimination (Katoh, 2017). In breast cancer, $\beta$-catenin can be translocated to the nucleus in response to substrate stiffness which may drive tumor progression through altering microRNA expression (Mouw et al., 2014). Guerra et al. (2017) found that modification of cells with the ROCK inhibitor, fasudil, caused $\beta$-catenin translocation to the nucleus. ROCK inhibition decreased breast cancer migration and also activated the canonical $\mathrm{Wnt} / \beta$ catenin pathway. Consequently, fasudil may be a candidate for preventing triple negative breast cancer metastasis and other treatments may be developed considering the role of translocation of proteins to the nucleus.

Finally, LINC complex-based nuclear mechanotransduction proteins, e.g., SUN1, SUN2, nesprin1/2, providing direct force links from the cytoskeleton to the nucleus may be important cancer markers, which are downregulated in breast cancer and may serve for diagnostic or prognostic purposes (Matsumoto et al., 2015). It was also recently reported that nesprin-2 and lamin $\mathrm{A} / \mathrm{C}$ were significantly decreased in malignant MCF-7 and MDA-MB-231 cells when tested on patterned substrates while these proteins were increased in benign MCF-10A cells, suggesting that the downregulation of LINC complex and nuclear lamina may have direct influence on cancer state and invasiveness (Antmen et al., 2019).

\section{PERSPECTIVE}

Mechanobiology is now well-posed to solve some of the most pertinent issues in cancer detection, grading, and typing. In this review paper, we highlighted the effects of mechanical factors in oncogenesis, the premetastatic niche, the metastatic cascade, colonization of secondary metastatic sites, and dormancy and drug resistance. Mechanotransduction pathways have been identified in the regulation of these processes, potentially suggesting treatment targets. Also, by testing mechanical effect on drug resistance, therapies may also be improved to increase the efficiency of current treatments. Besides sensitizing cells to chemotherapy drugs, understanding how to modify aberrant microenvironments for drug penetration may also be attempted. These works will require more biomimetic tumor models to understand cancer cell sensing and response to environmental cues and mechanical loading as well as the interaction with cancer-associated fibroblasts, luminal cells, and vascular cells. This perspective takes the cancer as tissue or organoid approach. Extending the soil-and-seed paradigm in the cancer research to the current understanding of cancer complexity in multiple domains, cancer may no longer be thought of as just a seed that takes root in favorable soil. Cancer is more akin to a seed that acts as a terraforming agent, capable of modifying local and system soil ecology. This is particularly evident when considering the mechanical factors of tumor progression. Modified ECM stiffness, chemical composition and physical substrate pattern, and transformation of endothelial, MSCs, and immune cells to tumor-supportive roles via mechanical loadings and mechanotransduction pathways associated thus reinforce the importance of considering the mechanical domains in tumor progression. Since both malignant and cancer-associated fibroblasts have demonstrated mechanical memory effect, model systems that mimic multiple steps of the metastatic cascade will be particularly useful, for example, studying intravasation and adherent migration after cells are conditioned in compressive environments, or studying the colonization of secondary tumor sites after mechanical loading exposure that mimics the invasion process.

In addition to therapies involving mechanical signaling or mechanotransduction pathways, classification of cancer and more accurate prognostics would benefit from considering the mechanical domain. Large heterogeneities in mechanical response still exist within individual cell lines and among cells with the same immune-profile (HER, ER, PR, etc.) or classification (luminal A, claudin low, etc.). Mechanotyping using measures of cytoskeletal stiffness, nuclear deformability, traction force, and adhesion profile could supplement existing classification methods and may help resolve heterogeneities present in the current classification system. For example, topography-based assessments may be effective for identifying malignant cells and distinguishing these form benign cells. Assessment of morphology and migration on different topographies may be used to screen for multiple salient features in cell behavior in a relatively short time (AlvarezElizondo et al., 2020). Guck et al. (2005) were able to interrogate the deformability of human mammary epithelial cells rapidly using a microfluidic setup with optically-induced surface tension without direct cell contact. The utility of this strategy has been demonstrated in oral cancer diagnosis with a low optical stress applied in the range of $1-5 \mathrm{~Pa}$ (Remmerbach et al., 2009). Applying a similar concept, forcing cells from blood samples quickly through a microfluidic channel allows the identification of cell types and detection of pathological changes via rheological analysis (Toepfner et al., 2018). Taking this a step further, dynamic typing via the response to mechanical loading regimes could be attempted. The activation of key mechanotransduction regulators to mechanical loadings would supplement the mechanotyping and current surface marker methods. This may necessitate the development of high throughput mechanical loading systems for such testing. Expanding on this, a novel device for combining multiple loading regimes, compression, fluid shear, and stretch, to be even more physiologically relevant will be helpful for interrogating tumor organoids. Current systems are typically limited to simple loading regimens and may not capture the complex and dynamic mechanical loading that exists in vivo.

\section{AUTHOR CONTRIBUTIONS}

BR and JL: manuscript planning, writing, and revision. EK and TB: manuscript revision and writing. All authors contributed to the article and approved the submitted version. 


\section{FUNDING}

We thank the funding supports given to JL: NIH/NIGMS Nebraska Center for the Prevention of Obesity Diseases (NPOD) Project Leader Grant (P20GM104320, PI:

\section{REFERENCES}

Acerbi, I., Cassereau, L., Dean, I., Shi, Q., Au, A., Park, C., et al. (2015). Human breast cancer invasion and aggression correlates with ECM stiffening and immune cell infiltration. Integr. Biol. 7, 1120-1134. doi: 10.1039/c5ib0 $0040 \mathrm{~h}$

Aguado, B. A., Hartfield, R. M., Bushnell, G. G., Decker, J. T., Azarin, S. M., Nanavati, D., et al. (2018). Biomaterial scaffolds as pre-metastatic niche mimics systemically alter the primary tumor and tumor microenvironment. Adv. Healthc. Mater. 7:e1700903. doi: 10.1002/adhm.201700903

Alcoser, T. A., Bordeleau, F., Carey, S. P., Lampi, M. C., Kowal, D. R., Somasegar, S., et al. (2015). Probing the biophysical properties of primary breast tumorderived fibroblasts. Cell. Mol. Bioeng. 8, 76-85. doi: 10.1007/s12195-0140360-9

Alvarez-Elizondo, M. B., Li, C. W., Marom, A., Tung, Y. T., Drillich, G., Horesh, Y., et al. (2020). Micropatterned topographies reveal measurable differences between cancer and benign cells. Med. Eng. Phys. 75, 5-12. doi: 10.1016/j.medengphy.2019.11.004

Ansaryan, S., Khayamian, M. A., Saghafi, M., Shalileh, S., Nikshoar, M. S., Abbasvandi, F., et al. (2019). Stretch induces invasive phenotypes in breast cells due to activation of aerobic-glycolysis-related pathways. Adv. Biosyst. 3:e1800294. doi: 10.1002/adbi.201800294

Antmen, E., Demirci, U., and Hasirci, V. (2019). Amplification of nuclear deformation of breast cancer cells by seeding on micropatterned surfaces to better distinguish their malignancies. Colloids Surf. B Biointerfaces. 183:110402. doi: 10.1016/j.colsurfb.2019.110402

Balachander, G. M., Balaji, S. A., Rangarajan, A., and Chatterjee, K. (2015). Enhanced metastatic potential in a $3 \mathrm{~d}$ tissue scaffold toward a comprehensive in vitro model for breast cancer metastasis. ACS Appl. Mater. Interfaces 7, 27810-27822. doi: 10.1021/acsami.5b09064

Balzer, E. M., Tong, Z., Paul, C. D., Hung, W. C., Stroka, K. M., Boggs, A. E., et al. (2012). Physical confinement alters tumor cell adhesion and migration phenotypes. FASEB J. 26, 4045-4056. doi: 10.1096/fj.12-211441

Berrueta, L., Bergholz, J., Munoz, D., Muskaj, I., Badger, G. J., Shukla, A., et al. (2018). Stretching reduces tumor growth in a mouse breast cancer model. Sci. Rep. 8, 1-7. doi: 10.1038/s41598-018-26198-7

Berrueta, L., Muskaj, I., Olenich, S., Butler, T., Badger, G. J., Colas, R. A., et al. (2016). Stretching impacts inflammation resolution in connective tissue. J. Cell. Physiol. 231, 1621-1627. doi: 10.1002/jcp.25263

Bouzid, T., Kim, E., Riehl, B. D., Esfahani, A. M., Rosenbohm, J., Yang, R., et al. (2019). The LINC complex, mechanotransduction, and mesenchymal stem cell function and fate. J. Biol. Eng. 13:68. doi: 10.1186/s13036-019-0197-9

Boyd, N. F., Li, Q., Melnichouk, O., Huszti, E., Martin, L. J., Gunasekara, A., et al. (2014). Evidence that breast tissue stiffness is associated with risk of breast cancer. PLoS ONE 9:e100937. doi: 10.1371/journal.pone.0100937

Bregenzer, M. E., Horst, E. N., Mehta, P., Novak, C. M., Raghavan, S., Snyder, C. S., et al. (2019). Integrated cancer tissue engineering models for precision medicine. PLoS ONE 14:e0216564. doi: 10.1371/journal.pone.0216564

Brückner, D. B., Fink, A., Rädler, J. O., and Broedersz, C. P. (2020). Disentangling the behavioural variability of confined cell migration. J. R. Soc. Interface 17:20190689. doi: 10.1098/rsif.2019.0689

Byrne, K. M., Monsefi, N., Dawson, J. C., Degasperi, A., Bukowski-Wills, J. C., Volinsky, N., et al. (2016). Bistability in the racl, pak, and rhoa signaling network drives actin cytoskeleton dynamics and cell motility switches. Cell. Syst. 2, 38-48. doi: 10.1016/j.cels.2016.01.003

Calvo, F., Ege, N., Grande-Garcia, A., Hooper, S., Jenkins, R. P., Chaudhry, S. I., et al. (2013). Mechanotransduction and YAP-dependent matrix remodelling is required for the generation and maintenance of cancer associated fibroblasts. Nat. Cell Biol. 15, 637-646. doi: 10.1038/ncb2756
Zempleni), NIH/NIGMS Great Plains IDeA-CTR Pilot Grant (1U54GM115458-01, PI: Rizzo), NSF grant (1826135, PI: Yang), AHA Scientist Development Grant (17SDG33680170, PI: Duan), and Nebraska Collaboration Initiative Team Strengthening Seed Grants (PI: JL).

Carey, S. P., Martin, K. E., and Reinhart-King, C. A. (2017). Three-dimensional collagen matrix induces a mechanosensitive invasive epithelial phenotype. Sci. Rep. 7:42088. doi: 10.1038/srep42088

Chen, M. B., Whisler, J. A., Jeon, J. S., and Kamm, R. D. (2013). Mechanisms of tumor cell extravasation in an in vitro microvascular network platform. Integr. Biol. 5, 1262-1271. doi: 10.1039/c3ib40149a

Chen, S., Hourwitz, M. J., Campanello, L., Fourkas, J. T., Losert, W., and Parent, C. A. (2019). Actin cytoskeleton and focal adhesions regulate the biased migration of breast cancer cells on nanoscale asymmetric sawteeth. ACS Nano 13, 1454-1468. doi: 10.1021/acsnano.8b07140

Choi, H. Y., Yang, G.-M., Dayem, A. A., Saha, S. K., Kim, K., Yoo, Y., et al. (2019). Hydrodynamic shear stress promotes epithelial-mesenchymal transition by downregulating ERK and GSK3 $\beta$ activities. Breast Cancer Res. 21:6. doi: 10.1186/s13058-018-1071-2

Clegg, J., Koch, M. K., Thompson, E. W., Haupt, L. M., Kalita-de Croft, P., and Bray, L. J. (2020). Three-dimensional models as a new frontier for studying the role of proteoglycans in the normal and malignant breast microenvironment. Front. Cell Dev. Biol. 8:569454. doi: 10.3389/fcell.2020.569454

Cognart, H. A., Viovy, J. L., and Villard, C. (2020). Fluid shear stress coupled with narrow constrictions induce cell type-dependent morphological and molecular changes in SK-BR-3 and MDA-MB-231 cells. Sci. Rep. 10:6386. doi: 10.1038/s41598-020-63316-w

Demou, Z. N. (2010). Gene expression profiles in 3D Tumor analogs indicate compressive strain differentially enhances metastatic potential. Ann. Biomed. Eng. 38, 3509-3520. doi: 10.1007/s10439-010-0097-0

DeSantis, C. E., Ma, J., Gaudet, M. M., Newman, L. A., Miller, K. D., Sauer, A. G., et al. (2019). Breast cancer statistics, 2019. CA Cancer J. Clin. 69, 438-451. doi: $10.3322 /$ caac. 21583

Devadas, D., Moore, T. A., Walji, N., and Young, E. W. K. (2019). A microfluidic mammary gland coculture model using parallel 3D lumens for studying epithelial-endothelial migration in breast cancer. Biomicrofluidics 13:064122. doi: $10.1063 / 1.5123912$

Dickinson, L. E., Lütgebaucks, C., Lewis, D. M., and Gerecht, S. (2012). Patterning microscale extracellular matrices to study endothelial and cancer cell interactions in vitro. Lab. Chip 12, 4244-4248. doi: 10.1039/c2lc40819h

Downey, C., Alwan, K., Thamilselvan, V., Zhang, L., Jiang, Y., Rishi, A. K., et al. (2006). Pressure stimulates breast cancer cell adhesion independently of cell cycle and apoptosis regulatory protein (CARP)-1 regulation of focal adhesion kinase. Am. J. Surg. 192, 631-635. doi: 10.1016/j.amjsurg.2006.08.006

DuChez, B. J., Doyle, A. D., Dimitriadis, E. K., and Yamada, K. M. (2019). Durotaxis by human cancer cells. Biophys. J. 116, 670-683. doi: 10.1016/j.bpj.2019.01.009

Er, E. E., Valiente, M., Ganesh, K., Zou, Y., Agrawal, S., Hu, J., et al. (2018). Pericytelike spreading by disseminated cancer cells activates yap and mrtf for metastatic Colonization. Nat. Cell Biol. 20, 966-978. doi: 10.1038/s41556-018-0138-8

Fan, Y., Jalali, A., Chen, A., Zhao, X., Liu, S., Teli, M., et al. (2020). Skeletal loading regulates breast cancer-associated osteolysis in a loading intensity-dependent fashion. Bone Res. 8:9. doi: 10.1038/s41413-020-0083-6

Ferruzzi, J., Sun, M., Gkousioudi, A., Pilvar, A., Roblyer, D., Zhang, Y., et al. (2019). Compressive remodeling alters fluid transport properties of collagen networks - implications for tumor growth. Sci. Rep. 9:17151. doi: 10.1038/s41598-019-50268-z

Ficorella, C., Vázquez, R. M., Heine, P., Lepera, E., Cao, J., Warmt, E., et al. (2019). Normal epithelial and triple-negative breast cancer cells show the same invasion potential in rigid spatial confinement. New J. Phys. 21:083016. doi: 10.1088/1367-2630/ab3572

Gomes, N., Berard, M., Vassy, J., Peyri, N., Legrand, C., and Fauvel-Lafeve, F. (2003). Shear stress modulates tumour cell adhesion to the endothelium. Biorheology 40, 41-45. 
Guck, J., Schinkinger, S., Lincoln, B., Wottawah, F., Ebert, S., Romeyke, M., et al. (2005). Optical deformability as an inherent cell marker for testing malignant transformation and metastatic competence. Biophys. J. 88, 3689-3698. doi: 10.1529/biophysj.104.045476

Guerra, F. S., Oliveira, R. G., Fraga, C. A. M., Mermelstein, C. D. S., and Fernandes, P. D. (2017). ROCK inhibition with fasudil induces beta-catenin nuclear translocation and inhibits cell migration of MDA-MB 231 human breast cancer cells. Sci. Rep. 7:13723. doi: 10.1038/s41598-017-14216-z

Guo, Y., Steele, H. E., Li, B.-Y., and Na, S. (2020). Fluid flow-induced activation of subcellular AMPK and its interaction with FAK and Src. Arch. Biochem. Biophys. 679:108208. doi: 10.1016/j.abb.2019.108208

Guzman, A., Sánchez Alemany, V., Nguyen, Y., Zhang, C. R., and Kaufman, L. J. (2017). A novel 3D in vitro metastasis model elucidates differential invasive strategies during and after breaching basement membrane. Biomaterials 115, 19-29. doi: 10.1016/j.biomaterials.2016.11.014

Haessler, U., Teo, J. C. M., Foretay, D., Renaud, P., and Swartz, M. A. (2012). Migration dynamics of breast cancer cells in a tunable 3D interstitial flow chamber. Integr. Biol. 4, 401-409. doi: 10.1039/c1ib00128k

Holliday, D. L., and Speirs, V. (2011). Choosing the right cell line for breast cancer research. Breast Cancer Res. 13:215. doi: 10.1186/bcr2889

Ishihara, S., Inman, D. R., Li, W. J., Ponik, S. M., and Keely, P. J. (2017). Mechanosignal transduction in mesenchymal stem cells induces prosaposin secretion to drive the proliferation of breast cancer cells. Cancer Res. 77, 6179-6189. doi: 10.1158/0008-5472.CAN-17-0569

Jabbari, E., Sarvestani, S. K., Daneshian, L., and Moeinzadeh, S. (2015). Optimum 3D matrix stiffness for maintenance of cancer stem cells is dependent on tissue origin of cancer cells. PLoS ONE 10:e0132377. doi: 10.1371 /journal.pone. 0132377

Jiménez-Torres, J. A., Virumbrales-Muñoz, M., Sung, K. E., Lee, M. H., Abel, E. J., and Beebe, D. J. (2019). Patient-specific organotypic blood vessels as an in vitro model for anti-angiogenic drug response testing in renal cell carcinoma. EBioMedicine 42, 408-419. doi: 10.1016/j.ebiom.2019.03.026

Jones, B. C., Kelley, L. C., Loskutov, Y. V., Marinak, K. M., Kozyreva, V. K., Smolkin, M. B., et al. (2017). Dual targeting of mesenchymal and amoeboid motility hinders metastatic behavior. Mol. Cancer Res. 15, 670-682. doi: 10.1158/1541-7786.MCR-16-0411

Kang, S. A., Blache, C. A., Bajana, S., Hasan, N., Kamal, M., Morita, Y., et al. (2016). The effect of soluble E-selectin on tumor progression and metastasis. BMC Cancer 16:331. doi: 10.1186/s12885-016-2366-2

Kang, S. A., Hasan, N., Mann, A. P., Zheng, W., Zhao, L., Morris, L., et al. (2015). Blocking the adhesion cascade at the premetastatic niche for prevention of breast cancer metastasis. Mol. Ther. 23, 1044-1054. doi: 10.1038/mt.2015.45

Kar, S., Molla, M. S., Katti, D. R., and Katti, K. S. (2019). Tissueengineered nanoclay-based $3 \mathrm{D}$ in vitro breast cancer model for studying breast cancer metastasis to bone. J. Tissue Eng. Regen. Med. 13, 119-130. doi: $10.1002 /$ term. 2773

Katoh, M. (2017). Canonical and non-canonical WNT signaling in cancer stem cells and their niches: cellular heterogeneity, omics reprogramming, targeted therapy and tumor plasticity (review). Int. J. Oncol. 51, 1357-1369. doi: 10.3892/ijo.2017.4129

Kim, B. G., Gao, M. Q., Kang, S., Choi, Y. P., Lee, J. H., Kim, J. E., et al. (2017). Mechanical compression induces VEGFA overexpression in breast cancer via DNMT3A-dependent miR-9 downregulation. Cell Death Dis. 8:e2646. doi: $10.1038 /$ cddis. 2017.73

Kim, B. G., Kang, S., Han, H. H., Lee, J. H., Kim, J. E., Lee, S. H., et al. (2016). Transcriptome-wide analysis of compression-induced microRNA expression alteration in breast cancer for mining therapeutic targets. Oncotarget 7 , 27468-27478. doi: 10.18632/oncotarget.8322

Kim, B. G., Sung, J. S., Jang, Y., Cha, Y. J., Kang, S., Han, H. H., et al. (2019). Compression-induced expression of glycolysis genes in CAFs correlates with EMT and angiogenesis gene expression in breast cancer. Commun. Biol. 2, 1-15. doi: 10.1038/s42003-019-0553-9

Kittur, H., Tay, A., Hua, A., Yu, M., and Di Carlo, D. (2017). Probing cell adhesion profiles with a microscale adhesive choice assay. Biophys. J. 113, 1858-1867. doi: 10.1016/j.bpj.2017.08.028

Knouse, K. A., Lopez, K. E., Bachofner, M., and Amon, A. (2018). Chromosome segregation fidelity in epithelia requires tissue architecture. Cell 175, 200-211.e13. doi: 10.1016/j.cell.2018.07.042
Lim, J. Y., and Donahue, H. J. (2007). Cell sensing and response to micro-and nanostructured surfaces produced by chemical and topographic patterning. Tissue Eng. 13, 1879-1891. doi: 10.1089/ten.2006.0154

Lin, C. H., Pelissier, F. A., Zhang, H., Lakins, J., Weaver, V. M., Park, C., et al. (2015). Microenvironment rigidity modulates responses to the HER2 receptor tyrosine kinase inhibitor lapatinib via YAP and TAZ transcription factors. Mol. Biol. Cell. 26, 3946-3953. doi: 10.1091/mbc.E15-07-0456

Liu, S., Goldstein, R. H., Scepansky, E. M., and Rosenblatt, M. (2009). Inhibition of rho-associated kinase signaling prevents breast cancer metastasis to human bone. Cancer Res. 69, 8742-8751. doi: 10.1158/0008-5472.CAN-09-1541

Lynch, M. E., Brooks, D., Mohanan, S., Lee, M. J., Polamraju, P., Dent, K., et al. (2013). In vivo tibial compression decreases osteolysis and tumor formation in a human metastatic breast cancer model. J. Bone Miner. Res. 28, 2357-2367. doi: $10.1002 /$ jbmr. 1966

Matsubara, M., and Bissell, M. J. (2016). Inhibitors of Rho kinase (ROCK) signaling revert the malignant phenotype of breast cancer cells in 3D context. Oncotarget 7, 31602-31622. doi: 10.18632/oncotarget.9395

Matsumoto, A., Hieda, M., Yokoyama, Y., Nishioka, Y., Yoshidome, K., Tsujimoto, M., et al. (2015). Global loss of a nuclear lamina component, lamin A/C, and LINC complex components SUN1, SUN2, and nesprin-2 in breast cancer. Cancer Med. 4, 1547-1557. doi: 10.1002/cam4.495

Matthews, H. K., Ganguli, S., Plak, K., Taubenberger, A. V., Win, Z., Williamson, M., et al. (2020). Oncogenic signaling alters cell shape and mechanics to facilitate cell division under confinement. Dev. Cell. 52, 563-573.e3. doi: 10.1016/j.devcel.2020.01.004

McGrail, D. J., Kieu, Q. M. N., Iandoli, J. A., and Dawson, M. R. (2015). Actomyosin tension as a determinant of metastatic cancer mechanical tropism. Phys. Biol. 12:026001. doi: 10.1088/1478-3975/12/2/026001

Mekhdjian, A. H., Kai, F., Rubashkin, M. G., Prahl, L. S., Przybyla, L. M., McGregor, A. L., et al. (2017). Integrin-mediated traction force enhances paxillin molecular associations and adhesion dynamics that increase the invasiveness of tumor cells into a three-dimensional extracellular matrix. Mol. Biol. Cell. 28, 1467-1488. doi: 10.1091/mbc.e16-09-0654

Mina, S. G., Huang, P., Murray, B. T., and Mahler, G. J. (2017). The role of shear stress and altered tissue properties on endothelial to mesenchymal transformation and tumor-endothelial cell interaction. Biomicrofluidics 11:044104. doi: 10.1063/1.4991738

Mouw, J. K., Yui, Y., Damiano, L., Bainer, R. O., Lakins, J. N., Acerbi, I., et al. (2014). Tissue mechanics modulate microRNA-dependent PTEN expression to regulate malignant progression. Nat. Med. 20, 360-367. doi: 10.1038/ nm.3497

Mpekris, F., Angeli, S., Pirentis, A. P., and Stylianopoulos, T. (2015). Stressmediated progression of solid tumors: effect of mechanical stress on tissue oxygenation, cancer cell proliferation and drug delivery. Biomech. Model. Mechanobiol. 14, 1391-1402. doi: 10.1007/s10237-015-0682-0

Narkhede, A. A., Crenshaw, J. H., Crossman, D. K., Shevde, L. A., and Rao, S. S. (2020). An in vitro hyaluronic acid hydrogel based platform to model dormancy in brain metastatic breast cancer cells. Acta Biomater. 107, 65-77. doi: 10.1016/j.actbio.2020.02.039

Narkhede, A. A., Crenshaw, J. H., Manning, R. M., and Rao, S. S. (2018). The influence of matrix stiffness on the behavior of brain metastatic breast cancer cells in a biomimetic hyaluronic acid hydrogel platform. J. Biomed. Mater. Res. A. 106, 1832-1841. doi: 10.1002/jbm.a.36379

Nasrollahi, S., Walter, C., Loza, A. J., Schimizzi, G. V., Longmore, G. D., and Pathak, A. (2017). Past matrix stiffness primes epithelial cells and regulates their future collective migration through a mechanical memory. Biomaterials 146, 146-155. doi: 10.1016/j.biomaterials.2017.09.012

Neve, R. M., Chin, K., Fridlyand, J., Yeh, J., Baehner, F. L., Fevr, T., et al. (2006). A collection of breast cancer cell lines for the study of functionally distinct cancer subtypes. Cancer Cell 10, 515-527. doi: 10.1016/j.ccr.2006.10.008

Novak, C. M., Horst, E. N., Taylor, C. C., Liu, C. Z., and Mehta, G. (2019). Fluid shear stress stimulates breast cancer cells to display invasive and chemoresistant phenotypes while upregulating PLAU in a 3D bioreactor. Biotechnol. Bioeng. 116, 3084-3097. doi: 10.1002/bit.27119

Omidvar, R., Tafazzoli-shadpour, M., Shokrgozar, M. A., and Rostami, M. (2014). Atomic force microscope-based single cell force spectroscopy of breast cancer cell lines: an approach for evaluating cellular invasion. J. Biomech. 47, 3373-3379. doi: 10.1016/j.jbiomech.2014.08.002 
Page, J. M., Merkel, A. R., Ruppender, N. S., Guo, R., Dadwal, U. C., Cannonier, S., et al. (2015). Matrix rigidity regulates the transition of tumor cells to a bone-destructive phenotype through integrin $\beta 3$ and TGF- $\beta$ receptor type II. Biomaterials 64, 33-44. doi: 10.1016/j.biomaterials.2015.06.026

Paszek, M. J., DuFort, C. C., Rossier, O., Bainer, R., Mouw, J. K., Godula, K., et al. (2014). The cancer glycocalyx mechanically primes integrin-mediated growth and survival. Nature 511, 319-325. doi: 10.1038/nature13535

Peela, N., Sam, F. S., Christenson, W., Truong, D., Watson, A. W., Mouneimne, G., et al. (2016). A three dimensional micropatterned tumor model for breast cancer cell migration studies. Biomaterials 81, 72-83. doi: 10.1016/j.biomaterials.2015.11.039

Plodinec, M., Loparic, M., Monnier, C. A., Obermann, E. C., Zanetti-Dallenbach, R., Oertle, P., et al. (2012). The nanomechanical signature of breast cancer. Nat. Nanotechnol. 7, 757-765. doi: 10.1038/nnano.2012.167

Polacheck, W. J., Charest, J. L., and Kamm, R. D. (2011). Interstitial flow influences direction of tumor cell migration through competing mechanisms. Proc. Natl. Acad. Sci. U.S.A. 108, 11115-11120. doi: 10.1073/pnas.1103581108

Polacheck, W. J., German, A. E., Mammoto, A., Ingber, D. E., and Kamm, R. D. (2014). Mechanotransduction of fluid stresses governs 3D cell migration. Proc. Natl. Acad. Sci. U.S.A. 111, 2447-2452. doi: 10.1073/pnas.1316848111

Poudel, I., Menter, D. E., and Lim, J. Y. (2012). Directing cell function and fate via micropatterning: role of cell patterning size, shape, and interconnectivity. Biomed. Eng. Lett. 2, 38-45. doi: 10.1007/s13534-012-0045-Z

Rao, S. S., Bushnell, G. G., Azarin, S. M., Spicer, G., Aguado, B. A., Stoehr, J. R., et al. (2016). Enhanced survival with implantable scaffolds that capture metastatic breast cancer cells in vivo. Cancer Res. 76, 5209-5218. doi: 10.1158/0008-5472.CAN-15-2106

Remmerbach, T. W., Wottawah, F., Dietrich, J., Lincoln, B., Wittekind, C., and Guck, J. (2009). Oral cancer diagnosis by mechanical phenotyping. Cancer Res. 69, 1728-1732. doi: 10.1158/0008-5472.CAN-08-4073

Riehl, B. D., Kim, E., Lee, J. S., Duan, B., Yang, R., Donahue, H. J., et al. (2020). The role of fluid shear and metastatic potential in breast cancer cell migration. J. Biomech. Eng. 142:101001. doi: 10.1115/1.4047076

Rubashkin, M., Cassereau, L., Bainer, R., DuFort, C., Yui, Y., Ou, G., et al. (2014). Force engages vinculin and promotes tumor progression by enhancing PI3kinase activation of phosphatidylinositol $(3,4,5)$-triphosphate. Cancer Res. 74, 4597-4611. doi: 10.1158/0008-5472.CAN-13-3698

Schwager, S. C., Bordeleau, F., Zhang, J., Antonyak, M. A., Cerione, R. A., and Reinhart-King, C. A. (2019). Matrix stiffness regulates microvesicleinduced fibroblast activation. Am. J. Physiol. Cell Physiol. 317, C82-C92. doi: 10.1152/ajpcell.00418.2018

Shahriyari, F., Janmaleki, M., Sharifi, S., Hesar, M. E., Hoshian, S., Taghiabadi, R., et al. (2020). Effect of cell imprinting on viability and drug susceptibility of breast cancer cells to doxorubicin. Acta Biomater. 113, 119-129. doi: 10.1016/j.actbio.2020.06.007

Smelser, A. M., Macosko, J. C., O’Dell, A. P., Smyre, S., Bonin, K., and Holzwarth, G. (2015). Mechanical properties of normal versus cancerous breast cells. Biomech. Model. Mechanobiol. 14, 1335-1347. doi: 10.1007/s10237-015-0677-x

Steele, H. E., Guo, Y., Li, B. Y., and Na, S. (2019). Mechanotransduction of mitochondrial AMPK and its distinct role in flow-induced breast cancer cell migration. Biochem. Biophys. Res. Commun. 514, 524-529. doi: 10.1016/j.bbrc.2019.04.191

Stowers, R. S., Allen, S. C., Sanchez, K., Davis, C. L., Ebelt, N. D., Van Den Berg, C., et al. (2016). Extracellular matrix stiffening induces a malignant phenotypic transition in breast epithelial cells. Cell. Mol. Bioeng. 10, 114-123. doi: 10.1007/s12195-016-0468-1

Stowers, R. S., Shcherbina, A., Israeli, J., Gruber, J. J., Chang, J., Nam, S., et al. (2019). Matrix stiffness induces a tumorigenic phenotype in mammary epithelium through changes in chromatin accessibility. Nat. Biomed. Eng. 3, 1009-1019. doi: 10.1038/s41551-019-0420-5

Stylianopoulos, T., Martin, J. D., Snuderl, M., Mpekris, F., Jain, S. R., and Jain, R. K. (2013). Coevolution of solid stress and interstitial fluid pressure in tumors during progression: implications for vascular collapse. Cancer Res. 73, 3833-3841. doi: 10.1158/0008-5472.CAN-12-4521

Takao, S., Taya, M., and Chiew, C. (2019). Mechanical stress-induced cell death in breast cancer cells. Biol. Open. 8:bio043133. doi: 10.1242/bio.043133

Tang, K., Li, S., Li, P., Xia, Q., Yang, R., Li, T., et al. (2020). Shear stress stimulates integrin $\beta 1$ trafficking and increases directional migration of cancer cells via promoting deacetylation of microtubules. Biochim. Biophys. Acta Mol. Cell. Res. 1867:118676. doi: 10.1016/j.bbamcr.2020.118676

Tarbell, J. M., and Cancel, L. M. (2016). The glycocalyx and its significance in human medicine. J. Intern. Med. 280, 97-113. doi: 10.1111/joim.12465

Tien, J., Truslow, J. G., and Nelson, C. M. (2012). Modulation of invasive phenotype by interstitial pressure-driven convection in aggregates of human breast cancer cells. PLoS ONE 7:e45191. doi: 10.1371/journal.pone.0045191

Toepfner, N., Herold, C., Otto, O., Rosendahl, P., Jacobi, A., Kräter, M., et al. (2018). Detection of human disease conditions by single-cell morphorheological phenotyping of blood. Elife 7:e29213. doi: 10.7554/eLife.29213

Triantafillu, U. L., Park, S., and Kim, Y. (2019). Fluid shear stress induces drug resistance to doxorubicin and paclitaxel in the breast cancer cell line mcf7. Adv. Ther. 2:1800112. doi: 10.1002/adtp.201800112

Tse, J. M., Cheng, G., Tyrrell, J. A., Wilcox-Adelman, S. A., Boucher, Y., Jain, R. K., et al. (2012). Mechanical compression drives cancer cells toward invasive phenotype. Proc. Natl. Acad. Sci. U.S.A. 109, 911-916. doi: 10.1073/pnas.1118910109

Tseng, Q., Wang, I., Duchemin-Pelletier, E., Azioune, A., Carpi, N., Gao, J., et al. (2011). A new micropatterning method of soft substrates reveals that different tumorigenic signals can promote or reduce cell contraction levels. Lab. Chip. 11, 2231-2240. doi: 10.1039/c0lc00641f

Wang, Y., Chen, Z., Xiao, L., Du, Z., Han, X., Yu, X., et al. (2012). Evaluating cell migration in vitro by the method based on cell patterning within microfluidic channels. Electrophoresis 33, 773-779. doi: 10.1002/elps.201100349

Wang, Y., Goliwas, K. F., Severino, P. E., Hough, K. P., Van Vessem, D., Wang, H., et al. (2020). Mechanical strain induces phenotypic changes in breast cancer cells and promotes immunosuppression in the tumor microenvironment. Lab. Invest. 100, 1503-1516. doi: 10.1038/s41374-020-0452-1

Wei, S. C., Fattet, L., Tsai, J. H., Guo, Y., Pai, V. H., Majeski, H. E., et al. (2015). Matrix stiffness drives epithelial-mesenchymal transition and tumour metastasis through a TWIST1-G3BP2 mechanotransduction pathway. Nat. Cell. Biol. 17, 678-688. doi: 10.1038/ncb3157

Wen, Z., Liang, Y., Deng, S., Zou, L., Xie, X., Yang, J., et al. (2019). Talin2 regulates invasion of human breast cancer MDA-MB-231 cells via alteration of the tumor microenvironment. Oncol. Lett. 17, 4835-4842. doi: 10.3892/ol.2019.10175

Wolfenson, H., Meacci, G., Liu, S., Stachowiak, M. R., Iskratsch, T., Ghassemi, S., et al. (2016). Tropomyosin controls sarcomere-like contractions for rigidity sensing and suppressing growth on soft matrices. Nat. Cell Biol. 18, 33-42. doi: $10.1038 / \mathrm{ncb} 3277$

Xin, Y., Chen, X., Tang, X., Li, K., Yang, M., Tai, W. C.-S., et al. (2019). Mechanics and actomyosin-dependent survival/chemoresistance of suspended tumor cells in shear flow. Biophys. J. 116, 1803-1814. doi: 10.1016/j.bpj.2019.04.011

Yadav, S., Vadivelu, R., Ahmed, M., Barton, M., and Nguyen, N.-T. (2019). Stretching cells - An approach for early cancer diagnosis. Exp. Cell. Res. 378, 191-197. doi: 10.1016/j.yexcr.2019.01.029

Yang, B., Wolfenson, H., Chung, V. Y., Nakazawa, N., Liu, S., Hu, J., et al. (2020). Stopping transformed cancer cell growth by rigidity sensing. Nat. Mater. 19, 239-250. doi: 10.1038/s41563-019-0507-0

Yang, W., Yu, H., Li, G., Wang, B., Wang, Y., and Liu, L. (2016). Regulation of breast cancer cell behaviours by the physical microenvironment constructed via projection microstereolithography. Biomater. Sci. 4, 863-870. doi: 10.1039/C6BM00103C

Zanotelli, M. R., Goldblatt, Z. E., Miller, J. P., Bordeleau, F., Li, J., VanderBurgh, J. A., et al. (2018). Regulation of ATP utilization during metastatic cell migration by collagen architecture. Mol. Biol. Cell 29, 1-9. doi: 10.1091/mbc.E17-01-0041

Zhang, J., Goliwas, K. F., Wang, W., Taufalele, P. V., Bordeleau, F., and ReinhartKing, C. A. (2019). Energetic regulation of coordinated leader-follower dynamics during collective invasion of breast cancer cells. Proc. Natl. Acad. Sci. U.S.A. 116, 7867-7872. doi: 10.1073/pnas.1809964116

Zhang, L., and Webster, T. J. (2012). Poly-lactic-glycolic-acid surface nanotopographies selectively decrease breast adenocarcinoma cell functions. Nanotechnology 23:155101. doi: 10.1088/0957-4484/23/15/155101

Zhang, L., and Webster, T. J. (2013). Effects of chemically modified nanostructured PLGA on functioning of lung and breast cancer cells. Int. J. Nanomed. 8, 1907-1919. doi: 10.2147/IJN.S41570

Zhang, X., and Lv, Y. (2017). Suspension state increases reattachment of breast cancer cells by up-regulating lamin A/C. Biochim. Biophys. Acta Mol. Cell Res. 1864, 2272-2282. doi: 10.1016/j.bbamcr.2017.09.006 
Zhang, X., Yang, L., Chien, S., and Lv, Y. (2018). Suspension state promotes metastasis of breast cancer cells by up-regulating cyclooxygenase- 2 . Theranostics 8, 3722-3736. doi: 10.7150/thno.25434

Zhao, L., Guo, T., Wang, L., Liu, Y., Chen, G., Zhou, H., et al. (2018). Tape-assisted photolithographic-free microfluidic chip cell patterning for tumor metastasis study. Anal. Chem. 90, 777-784. doi: 10.1021/acs.analchem. $7 \mathrm{~b} 03225$

Zhou, S. F., Gopalakrishnan, S., Xu, Y. H., To, S. K. Y., Wong, A. S. T., Pang, S. W., et al. (2017). Substrates with patterned topography reveal metastasis of human cancer cells. Biomed. Mater. 12:055001. doi: 10.1088/1748-605X/ aa785d

Zhu, P., Tseng, N. H., Xie, T., Li, N., Fitts-Sprague, I., Peyton, S. R., et al. (2019). Biomechanical microenvironment regulates fusogenicity of breast cancer cells. ACS Biomater. Sci. Eng. 5, 3817-3827. doi: 10.1021/acsbiomaterials. $8 \mathrm{~b} 00861$
Zhu, W., Holmes, B., Glazer, R. I., and Zhang, L. G. (2016). 3D printed nanocomposite matrix for the study of breast cancer bone metastasis. Nanomedicine 12, 69-79. doi: 10.1016/j.nano.2015.09.010

Conflict of Interest: The authors declare that the research was conducted in the absence of any commercial or financial relationships that could be construed as a potential conflict of interest.

Copyright (c) 2021 Riehl, Kim, Bouzid and Lim. This is an open-access article distributed under the terms of the Creative Commons Attribution License (CC BY). The use, distribution or reproduction in other forums is permitted, provided the original author(s) and the copyright owner(s) are credited and that the original publication in this journal is cited, in accordance with accepted academic practice. No use, distribution or reproduction is permitted which does not comply with these terms. 